\title{
Boundary-layer receptivity for a parabolic leading edge
}

\author{
By P. W. HAMMERTON $\dagger$ AND E.J. KERSCHEN \\ Department of Aerospace \& Mechanical Engineering, University of Arizona, Tucson, \\ AZ 85721, USA
}

(Received 10 July 1995 and in revised form 5 November 1995)

The effect of the nose radius of a body on boundary-layer receptivity is analyzed for the case of a symmetric mean flow past a body with a parabolic leading edge. Asymptotic methods based on large Reynolds number are used, supplemented by numerical results. The Mach number is assumed small, and acoustic free-stream disturbances are considered. The case of free-stream acoustic waves, propagating obliquely to the symmetric mean flow is considered. The body nose radius, $r_{n}$, enters the theory through a Strouhal number, $S=\omega r_{n} / U$, where $\omega$ is the frequency of the acoustic wave and $U$ is the mean flow speed. The finite nose radius dramatically reduces the receptivity level compared to that for a flat plate, the amplitude of the instability waves in the boundary layer being decreased by an order of magnitude when $S=0.3$. Oblique acoustic waves produce much higher receptivity levels than acoustic waves propagating parallel to the body chord.

\section{Introduction}

The receptivity process through which free-stream disturbances generate instability waves in boundary layers was first discussed by Morkovin (1969). The transfer of energy from the free-stream disturbance to the instability wave generally comes about through non-parallel mean flow effects, which may arise either in the leading-edge region, or in a localized region farther downstream in the boundary layer (Goldstein \& Hultgren 1989; Kerschen 1990).

Up to now, theoretical studies of leading-edge receptivity have been restricted to a semiinfinite, zero-thickness plate. Goldstein (1983) developed an asymptotic analysis for this problem; leading-edge receptivity coefficients for various free-stream disturbances were calculated by Goldstein et al. (1983) and Heinrich \& Kerschen (1989). However, aerodynamic bodies designed for subsonic flow generally have finite thickness distributions with a parabolic leading edge. In the present paper, we examine the influence of the thickness of a body on leading-edge receptivity. The body is assumed to be two-dimensional, with a symmetric cross-section and a parabolic leading edge. An asymptotic theory for the case of a symmetric mean flow is developed in this paper. Results are presented for receptivity to acoustic waves in the free stream, incident on the body at arbitrary angle. Attention is focused on the variation of the receptivity level with the nose radius of the body and the incidence angle of the acoustic field.

In $\S 2$, a high Reynolds number asymptotic analysis $\left(\epsilon^{6}=\nu \omega / U_{e}^{2} ; \epsilon \ll 1\right)$ is formulated for an incompressible, two-dimensional flow. This follows the approach of Goldstein (1983) but remains valid for a nose radius comparable to the free-stream disturbance

$\dagger$ Present address: School of Mathematics, University of East Anglia, Norwich, NR4 7TJ, UK 
length scale $U_{e} / \omega$. Here $U_{e}$ is an effective free-stream speed, defined in $\S 2$, which includes a correction to the free-stream velocity due to the flow perturbation created by downstream portions of the airfoil. Two streamwise regions enter the analysis, one region where the distance downstream is $O\left(U_{e} / \omega\right)$ and the disturbance is governed by the linearised unsteady boundary layer equation (LUBLE), and a second region at distances $O\left(\epsilon^{-2} U_{e} / \omega\right)$ where the disturbance is governed by the triple-deck structure, corresponding to the asymptotic form of the Orr-Sommerfeld equation (OSE) in the vicinity of the lower branch.

The structure of the solution in the LUBLE region is analysed in $\S 3$. The inviscid pressure field and slip velocity induced by the free-stream disturbance (analysed in $\S 4$ ) drives the unsteady motion in the boundary layer. Far downstream in the LUBLE region, the solution consists of a Stokes wave, and a set of asymptotic eigensolutions that contain velocity but not pressure fluctuations. These asymptotic eigensolutions are equivalent to those obtained by Lam \& Rott (1960) and Ackerberg \& Phillips (1972), but modified to include the effects of the mean pressure gradient and surface curvature. The asymptotic analysis for distances far downstream determines the form of the eigenfunctions, but not their coefficients $C_{i}$. These coefficients can be found only through a full solution of the LUBLE, which must be determined by numerical methods. Lam \& Rott (1993) have recently generalised their eigenfunctions to take account of arbitrary streamwise variation in the mean flow. The direct development in parabolic coordinates presented here is more convenient for our purposes, but it can be shown that our expressions for the eigenfunctions are in agreement with these more general results.

The wavelengths of the asymptotic eigenfunctions shorten progressively with distance downstream. Eventually, the self-induced pressure field associated with the displacement thickness of each asymptotic eigenfunction becomes significant, and the triple-deck structure replaces the LUBLE as the correct asymptotic approximation to the Navier-Stokes equation. The first asymptotic eigenfunction of the LUBLE matches on to the TollmienSchlicting wave solution of this triple-deck region. Thus, the form of the free-stream disturbance and the geometry close to the nose influence the amplitude of the TollmienSchlicting wave only through the coefficient $C_{1}$ of the first asymptotic eigenfunction. Therefore, we call $C_{1}$ the 'Receptivity Coefficient'. The primary objective of this paper is to determine the Receptivity Coefficient as a function of leading-edge geometry and free-stream disturbance characteristics.

For the flat-plate case, it is not clear whether the set of eigensolutions obtained by Lam \& Rott are complete. A second, very different set of eigensolutions was obtained by Brown \& Stewartson (1973) which, they argue, better represent the physical properties of the flow. While the relationship between these two sets is a fundamental question which deserves further study, this must first be done in the context of the flat-plate problem. For a parabolic body, generalisations of the Brown \& Stewartson eigensolutions should also exist, but we concentrate solely on the generalisations of the Lam-Rott solutions since it is demonstrated in Appendix B that they match naturally to the Orr-Sommerfeld modes further downstream.

In $\S 4$, the inviscid pressure field and slip velocity produced by the interaction of a freestream acoustic wave with an airfoil are determined. The exact form of the unsteady slip velocity in the vicinity of the leading edge is determined by the global solution about the airfoil. This depends on the magnitude of the reduced acoustic frequency $k=\omega b / c$, where $b$ is the airfoil semi-chord and $c$ is the speed of sound. Here we present results for the limiting cases $k \ll 1$ and $k \gg 1$, when relatively simple expressions for the slip velocity can be obtained. Numerical solutions of the LUBLE are then carried out in $\S 5$, and comparisons with the asymptotic eigenfunctions of $\S 3$ are utilised to determine 


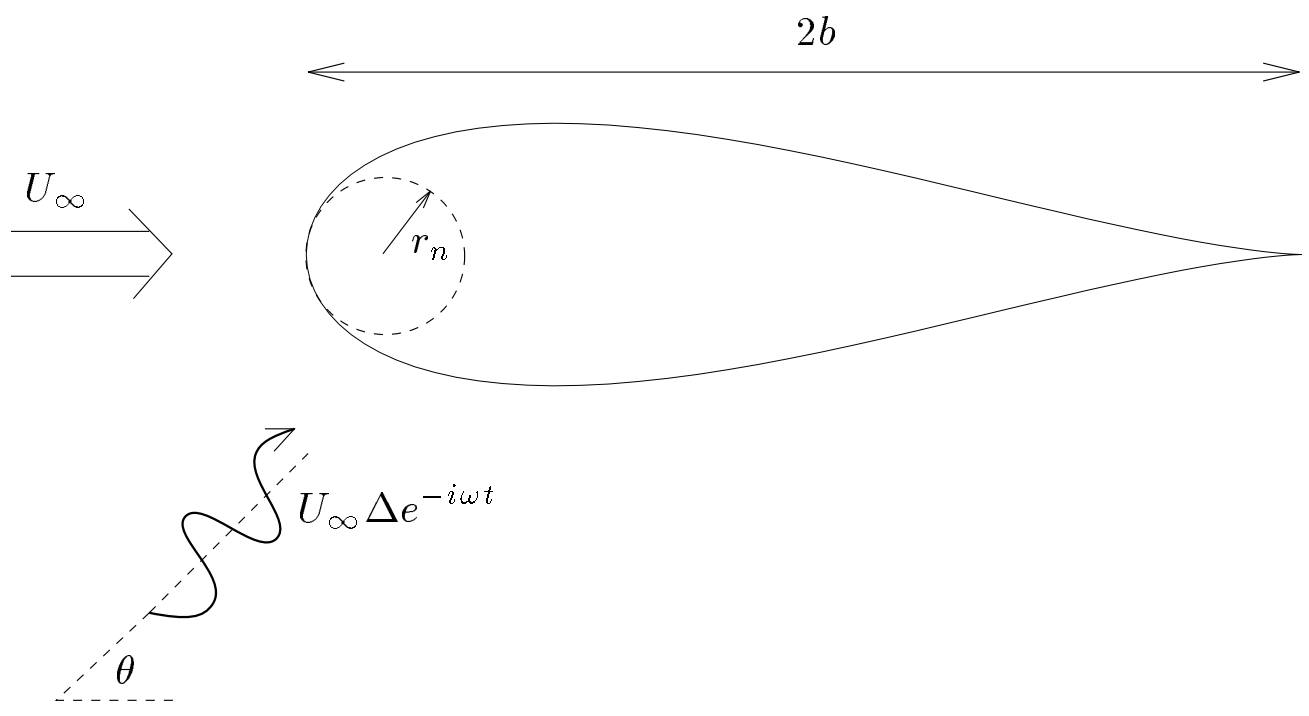

FIGURE 1. An illustration of the physical situation of interest: a thin, symmetric airfoil of chord $2 b$ is at zero angle-of-attack in a uniform flow of speed $U_{\infty}$, with a plane wave incident at an angle $\theta$ with respect to the airfoil chord.

the Receptivity Coefficient $C_{1}$ as a function of $S=\omega r_{n} / U_{e}$ and characteristics of the free-stream acoustic wave. The analysis presented is for $S=O(1)$; the only restriction is that $S \ll \epsilon^{-2}$, so that the unsteady disturbance in the nose region is governed by the LUBLE.

\section{Formulation}

We consider a thin, symmetric airfoil of chord $2 b$ at zero angle-of-attack in a uniform flow of speed $U_{\infty}$. A plane acoustic wave of frequency $\omega$, propagating at an angle $\theta$ with respect to the airfoil chord, is assumed to be incident on the airfoil as illustrated in figure 1. Two-dimensional, low-Mach-number flow is considered. Since the Mach number is small, the mean flow can be analyzed using incompressible theory. For the unsteady component of the flow, most features of interest can also be analyzed with incompressible theory. The influence of compressibility on the unsteady component of the flow is discussed in $\S 4$. The Reynolds number is assumed large, so the flow field is inviscid and irrotational everywhere except in the vicinity of the airfoil surface.

\subsection{Inviscid Outer Flow}

Introducing Cartesian coordinates $(x, y)$ normalized by the airfoil semi-chord $b$, with the origin located at the airfoil leading edge, the airfoil shape is given by

$$
y= \pm \delta s(x), \quad 0 \leq x \leq 2
$$

where $s(x)$ is the non-dimensional thickness distribution of the airfoil and the thickness parameter $\delta \ll 1$. The airfoil is assumed to have a rounded leading edge and a sharp trailing edge. The dimensional complex potential $W$ for the steady, inviscid flow past 
the airfoil is given by thin-airfoil theory,

$$
W=U_{\infty} b\left[z+\delta \frac{1}{\pi} \int_{0}^{2} \frac{\mathrm{d} s}{\mathrm{~d} x_{1}} \ln \left(z-x_{1}\right) \mathrm{d} x_{1}+O\left(\delta^{2}\right)\right],
$$

where $z=x+\mathrm{i} y$ and the dimensional velocity $(u, v)$ is given by $u-\mathrm{i} v=b^{-1} \mathrm{~d} W / \mathrm{d} z$.

For an airfoil with a rounded leading edge of radius $r_{n}$, the small argument expansion of the thickness distribution has the form

$$
s(x)=s_{1} x^{1 / 2}+s_{2} x+s_{3} x^{3 / 2}+O\left(x^{2}\right)
$$

where $s_{1}=\left(2 r_{n} / \delta^{2} b\right)^{1 / 2}$. The coefficient of the leading term in (2.3) must be $O(1)$, implying that $r_{n}=O\left(\delta^{2} b\right)$. The thin-airfoil expansion (2.2) is invalid in the vicinity of the leading edge, and must be replaced by a local expansion (Van Dyke, 1964a: Chapter 4). The natural length scale for this local expansion is the airfoil nose radius $r_{n}$. Introducing a parabolic coordinate system $(\bar{\xi}, \bar{\eta})$ in the local region, with $r_{n}$ as the length scale,

$$
x+\mathrm{i} y=\frac{1}{2} \frac{r_{n}}{b}\left[(\bar{\xi}+\mathrm{i} \bar{\eta})^{2}+1\right]
$$

the airfoil surface is given by $\bar{\eta}=1+\frac{1}{2} \delta s_{2} \bar{\xi}^{3} /\left(1+\bar{\xi}^{2}\right)+O\left(\delta^{2}\right)$. Thus, at leading order in the local coordinates, the airfoil surface is defined by the parabola $\bar{\eta}=1$.

The complex potential describing the leading approximation to the steady, inviscid flow in the vicinity of the airfoil nose is

$$
W=\frac{1}{2} U_{e} r_{n}(\bar{\xi}+i(\bar{\eta}-1))^{2},
$$

leading to a slip velocity, $U_{e} \bar{\xi} /\left(\bar{\xi}^{2}+1\right)^{1 / 2}$, where the constant $U_{e}$ is determined by matching with the thin airfoil expansion (2.2). For leading-edge shapes that contain a wedge component $\left(s_{2} \neq 0\right)$, the approximation $(2.5)$ is valid only at $O(1)$ in the thickness parameter $\delta$ and matching with (2.2) then shows that $U_{e}=U_{\infty}$. However, for leading-edge shapes in which the $S^{\frac{1}{2}}$ multiplies a locally analytic function of $x$, the even coefficients in (2.3) vanish. The Joukowski airfoil is one example of such an airfoil. In this case the leading-edge region is also parabolic at $O(\delta)$, and the matching then gives

$$
U_{e}=U_{\infty}+\frac{\delta}{\pi}\left\{\frac{s_{1}}{2^{\frac{1}{2}}}-\int_{0}^{2}\left[\frac{\mathrm{d} s}{\mathrm{~d} x}-\frac{s_{1}}{2 x^{\frac{1}{2}}}\right] \frac{d x}{x}\right\} .
$$

The $O(\delta)$ term in (2.6) is essentially a correction to the 'free-stream speed' in the local leading-edge region, due to the flow perturbation created by downstream portions of the airfoil.

The scattering of the acoustic wave by the airfoil produces an unsteady perturbation to the inviscid, irrotational flow described above. This unsteady, inviscid perturbation is considered in $\S 4$. The slip velocity and pressure associated with the inviscid flow drive the viscous flow in the boundary layer adjacent to the airfoil surface.

\subsection{Boundary-layer flow}

The boundary-layer flow in the vicinity of the leading edge is also analyzed most conveniently in parabolic coordinates. Although the nose radius $r_{n}$ is the most natural length scale for the steady flow, the length scale $U_{e} / \omega$ is more convenient for analysis of the unsteady flow. Thus, we introduce new coordinates,

$$
\tilde{\xi}=S^{\frac{1}{2}} \bar{\xi}, \quad \tilde{\eta}=S^{\frac{1}{2}} \bar{\eta}
$$


where

$$
S=\frac{\omega r_{n}}{U_{e}}
$$

is a Strouhal number based on the airfoil nose radius.

The flow around the body is analysed in terms of the incompressible vorticity equation. Utilising $U_{e}$ for the velocity scale, $U_{e} / \omega$ for the length scale and $\omega^{-1}$ for the time scale, the non-dimensional form of the vorticity equation is

$$
h^{2} \frac{\partial \tilde{\Omega}}{\partial t}+\frac{\partial(\tilde{\Omega}, \tilde{\Psi})}{\partial(\tilde{\xi}, \tilde{\eta})}=\epsilon^{6}\left(\frac{\partial^{2} \tilde{\Omega}}{\partial \tilde{\xi}^{2}}+\frac{\partial^{2} \tilde{\Omega}}{\partial \tilde{\eta}^{2}}\right)
$$

where $\tilde{\Psi}$ is the streamfunction, the velocity is given by $\mathbf{v}=h^{-1}\left(\tilde{\Psi}_{\tilde{\eta}} \mathbf{e}_{\tilde{\xi}}-\tilde{\Psi}_{\tilde{\xi}} \mathbf{e}_{\tilde{\eta}}\right)$, where $\mathbf{e}_{\tilde{\xi}}$ and $\mathbf{e}_{\tilde{\eta}}$ are unit vectors in the $\tilde{\xi}$ and $\tilde{\eta}$ coordinate directions, respectively, and the metric coefficient $h=\left(\tilde{\xi}^{2}+\tilde{\eta}^{2}\right)^{\frac{1}{2}}$. The vorticity $-\tilde{\Omega}$ is related to the stream function by $\tilde{\Omega}=h^{-2}\left(\tilde{\Psi}_{\tilde{\xi} \tilde{\xi}}+\tilde{\Psi}_{\tilde{\eta} \tilde{\eta}}\right)$. The small parameter

$$
\epsilon^{6}=\frac{\nu \omega}{U_{e}^{2}} \ll 1
$$

is the reciprocal of the Reynolds number based on the disturbance lengthscale. The flow satisfies the no-slip boundary conditions on the body surface,

$$
\tilde{\Psi}=\tilde{\Psi}_{\tilde{\eta}}=0, \quad \text { on } \quad \tilde{\eta}=S^{\frac{1}{2}},
$$

where terms of higher order in $\delta$ have been neglected. The analysis presented here considers the small- $\epsilon$ limit but with $S=O(1)$.

Since the Reynolds number is assumed large, viscosity is important only in a thin boundary layer adjacent to the body surface. To analyse the boundary-layer flow, we set

$$
\tilde{\eta}-S^{\frac{1}{2}}=\epsilon^{3} \eta, \quad \tilde{\xi}=\xi, \quad \tilde{\Psi}=\epsilon^{3} \Psi .
$$

The incompressible vorticity equation, expressed in terms of the stream function, then becomes

$$
\begin{aligned}
\Psi_{\eta \eta t}+\frac{\partial\left(\Psi_{\eta \eta} / H^{2}, \Psi\right)}{\partial(\xi, \eta)}-\frac{\Psi_{\eta \eta \eta \eta}}{H^{2}}=\epsilon^{6}( & \left.\frac{\Psi_{\eta \eta \xi \xi}}{H^{2}}+\left[\frac{\Psi_{\eta \eta}}{H^{2}}\right]_{\xi \xi}-\frac{\partial\left(\Psi_{\xi \xi} / H^{2}, \Psi\right)}{\partial(\xi, \eta)}-\Psi_{\xi \xi t}\right) \\
& +\epsilon^{12}\left[\frac{\Psi_{\xi \xi}}{H^{2}}\right]_{\xi \xi},
\end{aligned}
$$

where $H=\left(\xi^{2}+S\right)^{\frac{1}{2}}$. This equation is exact, except for the approximation that $h=H$ in the boundary layer. Careful consideration of the asymptotic structure, at all stages of the evolution of the disturbance, shows that the correction term $O\left(\epsilon^{3} \eta H^{-2}\right)$ never becomes significant and hence it is dropped throughout this presentation. At large values of $\eta$, the boundary-layer flow matches to the inviscid slip velocity,

$$
H^{-1} \Psi_{\eta} \rightarrow U_{s}(\xi, t), \quad \text { as } \quad \eta \rightarrow \infty .
$$

For $\mathrm{O}(1)$ values of $\xi$, the terms on the right side of (2.13) can be neglected, leading to the unsteady boundary-layer equation. The unsteady component of the flow, a small perturbation to the mean flow, then satisfies the linearised unsteady boundary layer equation (LUBLE). The LUBLE region is considered in the following section. However, the solution of the LUBLE contains components whose wavelengths progessively shorten with distance downstream. When $\xi=O\left(\epsilon^{-1}\right)$, terms on the right hand side of (2.13) 


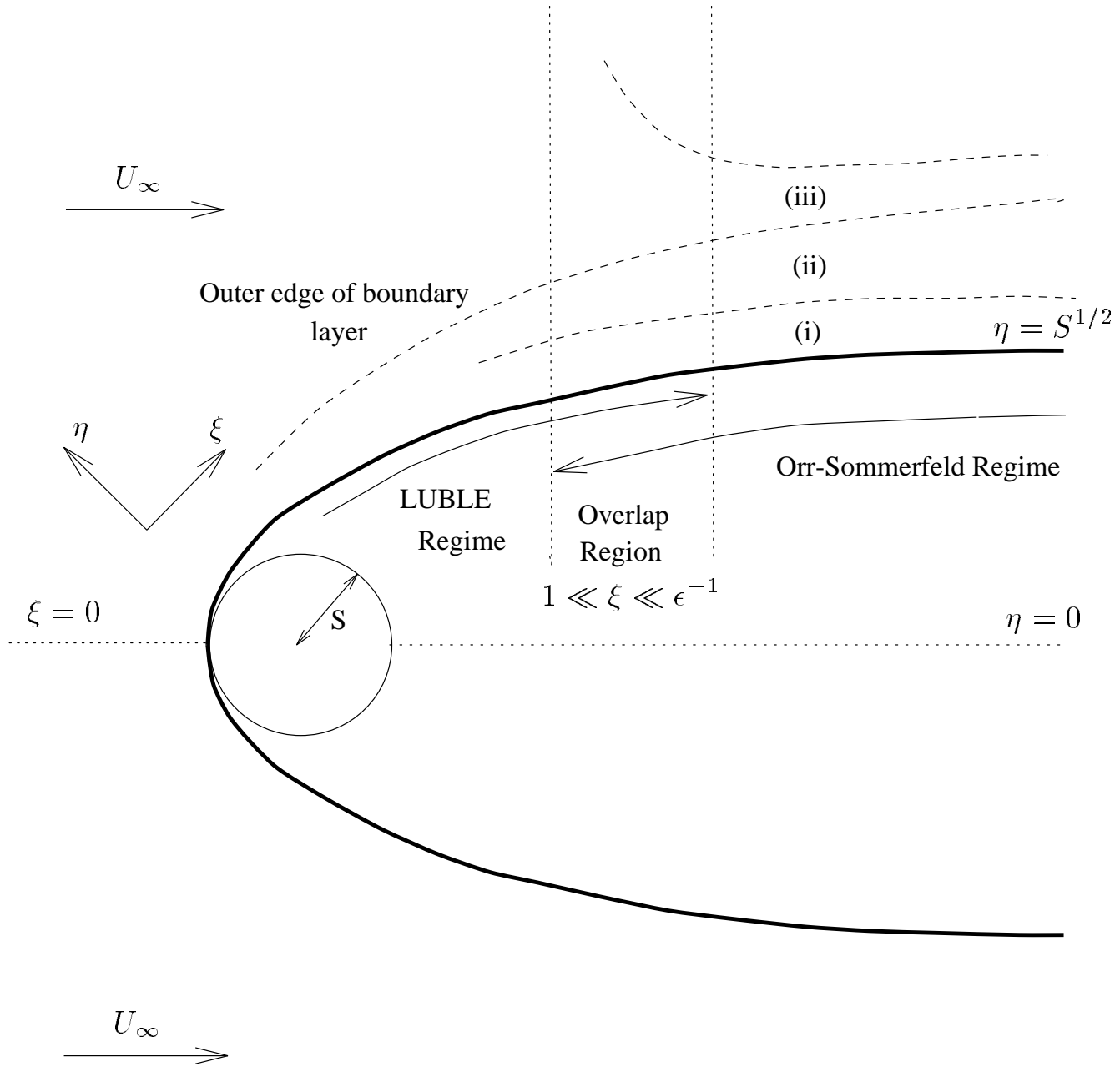

FiguRE 2. A schematic illustration of the boundary layer structure for a body with a parabolic leading-edge in a symmetric mean flow. The three decks in the Orr-Sommerfeld region are (i) the viscous wall layer; (ii) the main inviscid layer; and (iii) the outer irrotational layer.

become significant and the correct asymptotic approximation to (2.13) has the tripledeck structure. The structure of the developing boundary layer is summarised in figure 2. The asymptotic matching of the two streamwise regions is discussed briefly at the end of $\S 3$.

\section{Unsteady boundary-layer region}

In this section, we consider (2.13) for $\mathrm{O}(1)$ values of $\xi$ in the limit $\epsilon \rightarrow 0$. Subsequently, the behavior of this solution at large values of $\xi$ will be considered, in anticipation of matching with the Orr-Sommerfeld region that exists farther downstream in the boundary layer. For $\xi=O(1)$ and $\epsilon \rightarrow 0$, the terms on the right-hand side of (2.13) can be neglected. Integrating once with respect to $\eta$ then gives the unsteady boundary-layer 
equation

$$
H^{2} \Psi_{\eta t}+\left(\Psi_{\eta} \Psi_{\xi \eta}-\Psi_{\eta \eta} \Psi_{\xi}\right)-\xi H^{-2} \Psi_{\eta}^{2}-\Psi_{\eta \eta \eta}=-H^{2} P_{\xi}
$$

where the pressure gradient $P_{\xi}(\xi, t)$ is obtained by matching to the outer (inviscid) flow,

$$
P_{\xi}(\xi, t)=-H \frac{\partial U_{s}}{\partial t}-U_{s} \frac{\partial U_{s}}{\partial \xi} .
$$

In this paper, we consider uncambered airfoils at zero angle-of-attack to the mean flow. The slip velocity in the region of the parabolic nose is then given by

$$
U_{s}(\xi, t)=\frac{\xi}{H}+\Delta u_{s}(\xi) \mathrm{e}^{-\mathrm{i} t},
$$

where the steady contribution follows from (2.5), while the time-dependent perturbation depends on the particular form of the free-stream disturbance, as discussed in $\S 4$. Since the velocity field associated with an acoustic wave is of very small amplitude compared to the mean-flow speed, we assume that the amplitude scale factor $\Delta \ll 1$. The steady and unsteady components of the flow field can then be analysed separately. Thus, the streamfunction within the boundary layer can be written in the corresponding form

$$
\Psi=\xi \phi(\xi, \eta)+\Delta \psi(\xi, \eta) e^{-\mathrm{i} t} .
$$

where extraction of the factor $\xi$ from the mean-flow component is motivated by the form of (3.3). The function $\phi(\xi, \eta)$ describing the steady boundary-layer flow satisfies

$$
\phi_{\eta \eta \eta}+\phi_{\eta \eta} \phi+\xi\left(\phi_{\eta \eta} \phi_{\xi}-\phi_{\eta} \phi_{\eta \xi}\right)-S H^{-2}\left(\phi_{\eta}{ }^{2}-1\right)=0,
$$

with boundary conditions $\phi=\phi_{\eta}=0$ at $\eta=0$ and $\phi_{\eta} \rightarrow 1$ as $\eta \rightarrow \infty$. It may be observed that written in this form the steady equation involves $S$ and hence it appears that the steady flow is dependent on $\omega$, the frequency of the unsteady perturbation. However, $\omega$ also enters the scaling of the streamwise coordinate $\xi$. Re-writing (3.5) in terms of $\bar{\xi}=S^{-\frac{1}{2}} \xi$,

$$
\phi_{\eta \eta \eta}+\phi_{\eta \eta} \phi+\bar{\xi}\left(\phi_{\eta \eta} \phi_{\bar{\xi}}-\phi_{\eta} \phi_{\eta \bar{\xi}}\right)-\left(1+\bar{\xi}^{2}\right)^{-1}\left(\phi_{\eta}{ }^{2}-1\right)=0
$$

it is seen that the steady flow is indeed independent of $\omega$.

The time-dependent contribution to the boundary-layer flow satisfies the linearised unsteady boundary layer equation (LUBLE),

$$
\begin{gathered}
\mathcal{F}(\psi)=H^{2} \frac{\mathrm{d} p}{\mathrm{~d} \xi} \\
\mathcal{F}(\psi) \equiv \psi_{\eta \eta \eta}+\left(\phi+\xi \phi_{\xi}\right) \psi_{\eta \eta}+\left(\mathrm{i} H^{2}-\xi \phi_{\eta \xi}+\frac{\xi^{2}-S}{H^{2}} \phi_{\eta}\right) \psi_{\eta}+\xi\left(\phi_{\eta \eta} \psi_{\xi}-\phi_{\eta} \psi_{\eta \xi}\right) \\
\frac{\mathrm{d} p}{\mathrm{~d} \xi}=\left(\mathrm{i} H-\frac{S}{H^{3}}\right) u_{s}-\frac{\xi}{H} \frac{\partial u_{s}}{\partial \xi}
\end{gathered}
$$

with boundary conditions

$$
\psi=\psi_{\eta}=0 \quad \text { at } \quad \eta=0 \quad \text { and } \quad \psi_{\eta} \rightarrow H u_{s} \quad \text { as } \quad \eta \rightarrow \infty .
$$

These equations must be solved numerically; the methods used are described in $\S 5$. However, as discussed in $\S 1$, we are primarily interested in the component of the unsteady field that, in the large- $\xi$ limit, matches onto the Tollmien-Schlichting wave solution of the Orr-Sommerfeld equation. In the following two subsections, we develop large- $\xi$ asymptotic solutions for the steady and unsteady components of the flow. For convenience, in 
the rest of this section we restrict attention to the upper surface of the body (ie $\xi>0$ ). Corresponding results for $\xi<0$ can be obtained by inspection.

\subsection{Steady Boundary Layer Equation}

In order to analyse the large- $\xi$ behavior of the unsteady flow, the asymptotic form of the steady flow is required. The expansion for the steady flow is most naturally developed in terms of the streamwise coordinate scaled on the body nose radius, $\bar{\xi}$, as defined in (2.4). Far downstream of the nose $(\bar{\xi} \gg 1)$, the pressure gradient for the steady flow past a parabola decays to zero and hence the mean flow approaches the flat-plate solution. The asymptotic form of the solution is given by Van Dyke (1964b),

$$
\phi(\xi, \eta) \sim F(\eta)+A_{1} G_{1}(\eta) \frac{\ln \bar{\xi}^{2}}{\bar{\xi}^{2}}+\left(B_{1} G_{1}(\eta)+G_{2}(\eta)\right) \frac{1}{\bar{\xi}^{2}},
$$

where $F(\eta)$ is the Blasius function, $G_{1}(\eta)=\eta F^{\prime}(\eta)-F(\eta), A_{1}$ and $B_{1}$ are as yet undetermined numerical constants, and $G_{2}(\eta)$ satisfies

$$
G_{2}^{\prime \prime \prime}+F G_{2}^{\prime \prime}+2 F^{\prime} G_{2}^{\prime}-F^{\prime \prime} G_{2}=F^{\prime 2}-1+2 A_{1} F F^{\prime \prime}, \quad G_{2}(0)=G_{2}^{\prime}(0)=G_{2}^{\prime \prime}(0)=0 .
$$

The value of $A_{1}$ is fixed by the physical requirement of exponential decay of vorticity at the outer edge of the boundary layer. Hence $G_{2}(\eta)$ must decay exponentially for large $\eta$, which gives $A_{1}=0.60115$. The value of $B_{1}$ cannot be determined by the large- $\xi$ analysis since $\bar{\xi}^{-2} G_{1}(\eta)$ is an eigensolution of the perturbation equation. The next term in the expansion is $O\left(\bar{\xi}^{-\alpha}\right), \alpha \approx 3.774$, the fractional power arising as the next eigensolution of an infinite sequence (Libby \& Fox, 1963). It appears that $B_{1}$, together with the set of similar constants appearing in higher-order terms, is dependent on conditions close to the nose of the body and hence can be determined only by numerical integration from $\bar{\xi}=0$. This we discuss in $\S 5$.

The next four ignored terms in the expansion are of order $\bar{\xi}^{-3.774}, \bar{\xi}^{-4} \ln ^{2} \bar{\xi}, \bar{\xi}^{-4} \ln \bar{\xi}$ and $\bar{\xi}^{-4}$. As we will demonstrate in due course, the fact that these terms are of similar magnitude until $\bar{\xi}$ is extremely large poses certain problems in the numerical treatment of the problem.

In developing the solution to the LUBLE, the limiting behaviour of the steady flow close to the surface is required. This is found to be

$$
\phi \sim \frac{U_{0}^{\prime} \eta^{2}}{2}\left(1+A_{1} \frac{\ln \bar{\xi}^{2}}{\bar{\xi}^{2}}+B_{1} \frac{1}{\bar{\xi}^{2}}\right)-\frac{\eta^{3}}{6} \frac{1}{\bar{\xi}^{2}}-\frac{U_{0}^{\prime 2} \eta^{5}}{5 !}+O\left(\eta^{2} \bar{\xi}^{-3.774}, \eta^{5} \bar{\xi}^{-2} \ln \bar{\xi}\right),
$$

where $U_{0}^{\prime} \equiv F^{\prime \prime}(0)=0.4696$.

\subsection{Linearised Unsteady Boundary Layer Equation}

We now consider the evolution of the unsteady perturbation to the mean flow, which is governed by (3.7). Far downstream $(\xi \gg 1)$, the unsteady component of the streamfunction consists of a particular solution, $\psi_{p}$, determined entirely by the local conditions far downstream, together with a set of asymptotic eigensolutions,

$$
\psi(\eta, \xi ; S)=\psi_{p}(\eta, \xi ; S)+\sum_{i} C_{i}(S) \psi_{i}(\eta, \xi ; S) .
$$

The particular solution is a generalization of the classical Stokes layer solution, driven by the local value of the unsteady pressure gradient (3.7), see Lighthill (1954) for details. The eigensolutions $\psi_{i}$ depend on the geometry of the body far downstream, but are independent of the local free-stream disturbance. These eigensolutions are generalised forms of the eigenfunctions found by Lam \& Rott (1960) for the flat-plate boundary 
layer, taking account of the non-Blasius mean flow. The coefficients $C_{i}$ multiplying the asymptotic eigenfunctions are determined entirely by conditions close to the leading edge $(\xi=O(1))$. One of these eigensolutions, which we label $\psi_{1}$, matches on to the TollmienSchlichting wave in the Orr-Sommerfeld region farther downstream, where $\xi=O\left(\epsilon^{-1}\right)$. Thus, it is only through the coefficient $C_{1}$ that the unsteady disturbances in the free stream influence the amplitude of the Tollmien-Schlicting wave.

Our primary interest is in the relationship between the free-stream disturbances and the amplitude of the Tollmien-Schlichting wave. Thus, we focus on the asymptotic eigensolutions of the LUBLE. Guided by the results for the flat-plate case (Goldstein, 1983), we anticipate the development of a two-layer structure for $\xi \gg 1$, consisting of a main layer where $\eta=O(1)$ and a new inner layer of width $O\left(\xi^{-1}\right)$.

\subsubsection{Inner layer}

Introducing an inner variable $m=\xi \eta$, the homogeneous form of the LUBLE then becomes

$$
\begin{gathered}
\psi_{m m m}+\mathrm{i}\left(1+\frac{S}{\xi^{2}}\right) \psi_{m}+\frac{U_{0}^{\prime} m}{2 \xi^{3}}\left(2 \psi_{m}-m \psi_{m m}\right)+O\left(\frac{\ln \xi}{\xi^{5}}\right) \\
= \\
\frac{1}{\xi^{2}}\left\{U_{0}^{\prime} \gamma\left(\xi / S^{\frac{1}{2}}\right)\left(m \psi_{m \xi}-\psi_{\xi}\right)-\frac{S m}{2 \xi^{3}}\left(m \psi_{m \xi}-2 \psi_{\xi}\right)-\frac{U_{0}^{\prime 2} m^{3}}{24 \xi^{3}}\left(m \psi_{m \xi}-4 \psi_{\xi}\right)\right\}
\end{gathered}
$$

where it follows from (3.9) that $\gamma(\bar{\xi})=1+2 A_{1} \ln \bar{\xi} / \bar{\xi}^{2}+B_{1} / \bar{\xi}^{2}+O\left(\bar{\xi}^{-3.774}\right)$. The regular perturbation solution to (3.13), for large $\xi$, leads to solutions related to the particular solution $\psi_{p}$. Thus, the asymptotic eigensolutions must arise as a balance between the highest $m$ derivative (i.e. wall-normal derivative) and the terms involving $\xi$ derivatives. It is then clear that the eigensolution must contain a factor $\exp (T(\xi))$ and it is for this reason that the higher order terms containing derivatives with respect to $\xi$ have been retained in (3.13).

Writing $\psi=\exp (T(\xi)) f(\xi, M)$, where a new boundary layer variable $M=m(1+$ $\left.S / 2 \xi^{2}\right)$ has been introduced to simplify the solution, $f(\xi, M)$ satisfies

$$
f_{M M M}+i f_{M}-\frac{U_{0}^{\prime} \Gamma(\xi / \sqrt{S})}{\xi^{2}} \frac{\mathrm{d} T}{\mathrm{~d} \xi}\left(M f_{M}-f\right)=\frac{1}{\xi^{3}} R+O\left(\frac{1}{\xi^{4}}\right),
$$

where $\Gamma(\bar{\xi})=\gamma(\bar{\xi})-3 / 2 \bar{\xi}^{2}$ and

$$
\begin{aligned}
R=U_{0}^{\prime} \xi\left(M f_{M \xi}-f_{\xi}\right)+\frac{U_{0}^{\prime}}{2}\left(M^{2} f_{M M}-2 M f_{M}\right) \\
-\frac{U_{0}^{\prime 2} M^{3}}{4 ! \xi^{2}} \frac{\mathrm{d} T}{\mathrm{~d} \xi}\left(M f_{M}-4 f\right)-\frac{S M}{2 \xi^{2}} \frac{d T}{d \xi}\left(M f_{M}-2 f\right) .
\end{aligned}
$$

If $\mathrm{d} T / \mathrm{d} \xi$ is set equal to $-\lambda \xi^{2} / U_{0}^{\prime} \Gamma$, where $\lambda$ is an eigenvalue to be determined subsequently, solution to the leading order equation immediately follows using separation of variables,

$$
\psi \sim D \xi^{2 \tau} \mathrm{e}^{T(\xi)}\left[p(M)+\xi^{-3} q(M)+\ldots\right]
$$

Here $D$ is an arbitrary constant whose value is chosen for convenience later, and $\tau$ is a constant that is determined at a later stage of the analysis. The exponent $T(\xi)$ is then determined by integrating the large- $\xi$ expansion of $\xi^{2} / \Gamma\left(\xi / S^{\frac{1}{2}}\right)$. This exponent can be 
separated into two components, $T=T^{(0)}+T^{(1)}$, where

$$
T^{(0)}(\xi)=-\frac{\lambda \xi^{3}}{U_{0}^{\prime}}\left\{\frac{1}{3}-A_{1} S \frac{\ln \left(\xi^{2} / S\right)}{\xi^{2}}+\left(2 A_{1}+3 / 2-B_{1}\right) \frac{S}{\xi^{2}}\right\}
$$

is large for large $\xi$ and hence must be included in the leading-order form of the eigensolution, while the terms in $T^{(1)}(\xi)$ are small for $\xi \gg 1$ and thus could be considered as algebraic correction terms rather than as exponential amplitude functions. The first few terms in $T^{(1)}$ are of the form

$$
T^{(1)}(\xi)=b_{1} S^{1.887} \frac{1}{\xi^{0.774}}+b_{2} S^{2} \frac{\ln ^{2}\left(\xi^{2} / S\right)}{\xi}+b_{3} S^{2} \frac{\ln \left(\xi^{2} / S\right)}{\xi}+b_{4} S^{2} \frac{1}{\xi}+O\left(\xi^{-2.635}\right),
$$

where the coefficients $b_{i}$ are determined by the higher order terms of (3.11).

The leading-order mode-shape function $p(M)$ satisfies the differential equation

$$
\mathcal{L}(p)=0,
$$

where the operator $\mathcal{L}$ is defined by

$$
\mathcal{L}(p) \equiv p^{\prime \prime \prime}+\mathrm{i} p^{\prime}+\lambda\left(M p^{\prime}-p\right),
$$

with $p^{\prime} \equiv p_{M}$, etc. The boundary conditions at the wall are

$$
p(0)=p^{\prime}(0)=0,
$$

and matching to the main layer requires that $p^{\prime \prime} \rightarrow 0$ as $M \rightarrow \infty$ so as to avoid exponential growth of the inner solution. The differential operator and boundary conditions are homogenous and thus form an eigenvalue problem for $\lambda$. In fact, the leading-order equation is identical to the flat-plate equation, the effect of curvature having been absorbed into the boundary-layer variable $M$. Thus $p(M)$ and the value of the eigenvalue $\lambda=\mathrm{e}^{-\frac{1}{4} \mathrm{i} \pi} \rho^{-\frac{3}{2}}$ can be obtained immediately from Goldstein (1983) as

$$
p(M)=\frac{U_{0}^{\prime} \int_{0}^{M}(M-\tilde{M}) \operatorname{Ai}(\tilde{z}) \mathrm{d} \tilde{M}}{\int_{0}^{\infty} \operatorname{Ai}(\tilde{z}) \mathrm{d} \tilde{M}}, \quad \tilde{z}=\mathrm{e}^{\frac{1}{4} \mathrm{i} \pi} \rho^{-\frac{1}{2}} \tilde{M}-\rho,
$$

where $\rho$ is a solution of $\operatorname{Ai}^{\prime}(-\rho)=0$. An infinite set of such roots $\left(\rho_{i}>0\right)$ exists, with corresponding mode-shape functions $p_{i}(M)$.

The value of $\tau$ for each eigensolution, $\psi$, is then determined by a solvability condition on the $O\left(\xi^{-3}\right)$ correction to the mode shape, $q(M)$. This function satisfies

$$
\mathcal{L}(q)=\mathcal{R}(p),
$$

where

$$
\mathcal{R}(p) \equiv 2 \tau U_{0}^{\prime}\left(M p^{\prime}-p\right)+\frac{U_{0}^{\prime}}{2}\left(M^{2} p^{\prime \prime}-2 M p^{\prime}\right)-\frac{U_{0}^{\prime} M^{3}}{4 !}\left(M p^{\prime}-4 p\right)+\frac{S \lambda M}{2 U_{0}^{\prime}}\left(M p^{\prime}-2 p\right) .
$$

The boundary conditions at the wall and the matching condition to the main layer are the same as for $p(M)$. Integrating by parts and using the boundary/matching conditions on $p$ and $q$ gives the relation

$$
\int_{0}^{\infty} p^{\prime \prime \prime} \mathcal{L}(q) \mathrm{d} M=\int_{0}^{\infty} q^{\prime \prime \prime} \mathcal{L}(p) \mathrm{d} M,
$$

the right hand side vanishing by virtue of (3.19). Hence $\tau$ is determined by the condition

$$
\int_{0}^{\infty} p^{\prime \prime \prime} \mathcal{R}(p) \mathrm{d} M=0 .
$$


This gives $\tau=\tau^{(0)}+S \tau^{(1)}$, where $\tau^{(0)}$ is the flat-plate value and

$$
\tau^{(1)}=-\frac{\mathrm{e}^{-\frac{1}{4} \mathrm{i} \pi}}{4 U_{0}^{\prime 2} \rho^{\frac{3}{2}}} \frac{\int_{0}^{\infty} M p^{\prime \prime \prime}\left(M p^{\prime}-2 p\right) \mathrm{d} M}{\int_{0}^{\infty} p^{\prime \prime \prime}\left(M p^{\prime}-p\right) \mathrm{d} M} .
$$

It should be noted that this result does not involve linearisation in $S$, but is valid for $S=O(1)$. By suitable change of variables, this can be re-cast into the form

$$
\begin{aligned}
\tau^{(1)} & =\frac{\mathrm{i}}{4 U_{0}^{\prime 2} \rho} \frac{J_{2}-2 \mathrm{Ai}^{2}(-\rho)}{J_{1}}, \\
& =\frac{8 \rho^{3}-27}{40 U_{0}^{\prime 2} \rho^{3}} \mathrm{i}
\end{aligned}
$$

where expressions for the integrals $J_{n}=\int_{-\rho}^{\infty}(z+\rho)^{n}[\operatorname{Ai}(z)]^{2} \mathrm{~d} z$ are given in Appendix A. Similarly, the expression for $\tau^{(0)}$ (Goldstein, 1983) can be expressed in terms of $\rho$, avoiding the need for numerical evaluation of the integrals,

$$
\tau^{(0)}=\frac{1}{4}\left(-3+\frac{1}{12} \frac{J_{4}}{J_{1}}\right)=-\frac{889-16 \rho^{3}}{1260} .
$$

The value of $\tau_{i}$ for each eigensolution is obtained by substituting $\rho_{i}$ into (3.28) and (3.29). Finally, we set the value of the arbitrary constant in (3.16) to $D=2^{-\tau}$ so that, in the limit $S \rightarrow 0$, our expression for the asymptotic eigenfunction reduces to the result given by Goldstein (1983).

\subsubsection{Main layer}

In the main part of the boundary layer, where $\eta=O(1)$, the unsteady motion is essentially an inviscid response, driven by the displacement thickness of the inner layer. Setting $\psi=\left(\xi^{2} / 2\right)^{\tau} \exp (T(\xi)) g(\xi, \eta)$ and substituting into the homogeneous form of the LUBLE, $g(\xi, \eta)$ is found to satisfy

$$
\phi_{\eta \eta} g-\phi_{\eta} g_{\eta}=\mathrm{e}^{\frac{1}{4} \mathrm{i} \pi} \rho^{\frac{3}{2}} U_{0}^{\prime}\left(\frac{\mathrm{i} g_{\eta}}{\xi}+\frac{\left(\phi_{\eta \eta} g_{\xi}-\phi_{\eta} g_{\eta \xi}\right)}{\xi^{2}}\right)+O\left(\xi^{-3}\right) .
$$

Solving, and matching to the inner layer then gives

$$
g=\xi\left(F^{\prime}(\eta)+\frac{\mathrm{e}^{\frac{3}{4} \mathrm{i} \pi} \rho^{\frac{3}{2}} U_{0}^{\prime}}{\xi}+O\left(\xi^{-2}\right)\right) .
$$

\subsubsection{Large- $\xi$ matching}

In Appendix B it is shown that it is the first asymptotic eigensolution which matches on to the Tollmien-Schlichting wave that becomes unstable farther downstream. In $\S 5$, numerical solutions of the LUBLE are compared to the first asymptotic eigenfunction, in order to extract the receptivity coefficient $C_{1}$. The two most convenient points of comparison are the wall shear and the displacement thickness of the boundary layer. The wall shear is determined by the solution in the inner layer. However, for comparison with numerical solutions, it is most convenient to express the wall shear in terms of a derivative with respect to $\eta$. Rewriting (3.16) in terms of $\eta$ and evaluating $\psi_{\eta \eta}$ at $\eta=0$, the contribution to the wall shear due to the asymptotic eigensolution $\psi_{i}$ is

$$
\psi_{i}^{\prime \prime}(\eta=0)=\left(\frac{\xi^{2}}{2}\right)^{\tau_{i}} \mathrm{e}^{T_{i}(\xi)} \xi^{2} \frac{U_{0}^{\prime} \rho_{i}^{-\frac{1}{2}} \mathrm{e}^{\frac{1}{4} \mathrm{i} \pi}}{\int_{0}^{\infty} \operatorname{Ai}\left(z-\rho_{i}\right) d z}\left(1+O\left(\xi^{-2}\right)\right) .
$$


Taking the first root of $\operatorname{Ai}^{\prime}(-\rho)=0$, namely $\rho_{1}=1.01879$, and evaluating the numerical coefficient, the wall shear for the first asymptotic eigensolution is

$$
\psi_{1}^{\prime \prime}(\eta=0)=0.4356(1+\mathrm{i}) \mathrm{e}^{T_{1}^{(0)}(\xi)}\left(\frac{\xi^{2}}{2}\right)^{\tau_{1}+1} \mathrm{e}^{T_{1}^{(1)}(\xi)}\left(1+O\left(\xi^{-2}\right)\right),
$$

where $T_{1}^{(0)}(\xi)$ is given by $(3.17), T_{1}^{(1)}(\xi)$ is a small correction term given by (3.18) and

$$
\tau_{1}=-0.69213-S 1.9878 \mathrm{i}
$$

The contribution to the diplacement thickness due to an asymptotic eigensolution is obtained from the solution in the main layer,

$$
\psi_{i}(\eta \rightarrow \infty) \sim\left(\frac{\xi^{2}}{2}\right)^{\tau_{i}} \mathrm{e}^{T_{i}(\xi)} \xi\left(1+\frac{\mathrm{e}^{\frac{3}{4} \mathrm{i} \pi} \rho_{i}^{\frac{3}{2}} U_{0}^{\prime}}{\xi}+O\left(\xi^{-2}\right)\right),
$$

where the result for the first asymptotic eigensolution $\psi_{1}$ follows by substituting $\rho_{1}$ and $\tau_{1}$.

Since $T \propto \xi^{3}$ at leading order, the wavelengths of the asymptotic eigenfunctions decrease with distance downstream, increasing the importance of longitudinal derivatives. The LUBLE (3.1) is obtained from (2.13) by neglecting higher-order derivatives with respect to $\xi$. Thus, although the asymptotic eigensolutions derived in this section are uniformly valid solutions of the LUBLE as $\xi \rightarrow \infty$, they are not uniformly valid solutions of the full linearised Navier-Stokes equation.

Terms on the right side of (2.13), which were neglected in the LUBLE, become significant when $\xi=O\left(\epsilon^{-1}\right)$. An irrotational layer outside the mean boundary layer, driven by the oscillating displacement thickness of the eigensolutions, must then be considered, and the associated pressure gradient appears in the leading-order equations governing the inner layer. This coupled viscous-inviscid interaction has the triple-deck structure, corresponding to the small- $\epsilon$ asymptotic approximation to the Orr-Sommerfeld equation in the vicinity of the lower branch. The development of the boundary layer structure was summarised in figure 2. A complete treatment of the linear development of the instability would require an asymptotic solution for the Tollmien-Schlichting wave of the Orr-Sommerfeld equation, taking account of the surface curvature and non-zero pressure gradient. This Tollmien-Schlichting wave solution could then be matched to the first Lam-Rott asymptotic eigensolution of the LUBLE, as was done by Goldstein (1983) for the flat-plate case. However, for $O(1)$ values of $S$, with $\epsilon$ and $\delta$ of the same order, the Orr-Sommerfeld region is influenced by the full airfoil thickness distribution, necessitating a general development that does not seem justified in the present context. For flow around a semi-infinite parabolic body, we verify in Appendix B that the eigensolutions obtained here do indeed match on to the Tollmien-Schlichting wave.

In this paper we restrict ourselves to examining only the receptivity process, that is, the determination of the coefficient $C_{1}$ of the first eigensolution, which matches to the unstable Tollmien-Schlichting mode. The remainder of the paper is concerned with obtaining the Receptivity Coefficient for different free-stream disturbances. This is accomplished by comparing numerical solutions with the asymptotic solutions obtained above.

\section{Free-stream Disturbances}

In this section we consider the inviscid flow field produced by the interaction of a freestream acoustic wave with the airfoil. The acoustic wave is assumed to be incident on the airfoil at an angle $\theta$ with respect to the airfoil chord, as illustrated in figure 1 . The 
slip velocity and surface pressure fields generated by this interaction drive the unsteady motion in the boundary layer, leading to the generation of a Tollmien-Schlicting wave.

For a low Mach number flow, the acoustic wavelength $2 \pi c / \omega$ is long compared to the hydrodynamic length scale $U_{\infty} / \omega$. Thus, outside the boundary layer, the unsteady flow in the vicinity of the leading edge is incompressible and irrotational. Potential flow theory then shows that this local flow has the form

$$
u_{s}(\xi)=\kappa_{s}(\theta) \frac{\xi}{H}+\kappa_{a}(\theta) \frac{1}{H} .
$$

Here $\xi / H$ and $1 / H$ correspond to purely symmetric and anti-symmetric flow about the leading edge, respectively. The coefficients $\kappa_{s}$ and $\kappa_{a}$ multiplying these eigenfunctions are independent of the nose geometry, but depend on the free-stream disturbance, being determined by global features of the unsteady flow. Substituting (4.1) into (3.7) gives

$$
\mathcal{F}(\psi)=\kappa_{s} \xi\left(\mathrm{i} H^{2}-\frac{2 S}{H^{2}}\right)+\kappa_{a}\left(\mathrm{i} H^{2}+\frac{\xi^{2}-S}{H^{2}}\right) .
$$

Hence, writing $\psi=\kappa_{s} \psi_{s}+\kappa_{a} \psi_{a}$, it follows that the Receptivity Coefficient for the upper surface is given by

$$
C_{1}(S)=\kappa_{s}(\theta) C_{s}(S)+\kappa_{a}(\theta) C_{a}(S),
$$

where $C_{s}$ and $C_{a}$ are extracted from the solutions of

$$
\left.\begin{array}{l}
\mathcal{F}\left(\psi_{s}\right)=\xi\left(\mathrm{i} H^{2}-\frac{2 S}{H^{2}}\right) \\
\mathcal{F}\left(\psi_{a}\right)=\mathrm{i} H^{2}+\frac{\xi^{2}-S}{H^{2}}
\end{array}\right\}
$$

respectively. The corresponding Receptivity Coefficient for the lower surface is obtained by replacing $\theta$ by $-\theta$.

The remainder of this section is concerned with calculating $\kappa_{s}(\theta)$ and $\kappa_{a}(\theta)$, the coefficients of symmetric and anti-symmetric flow about the nose. These coefficients are found by asymptotic matching of the local solution (4.1) for the unsteady slip velocity with an appropriate global solution. The nature of this solution depends upon the magnitude of the reduced acoustic frequency $k=\omega b / c$, where $c$ is the speed of sound in the undisturbed medium. Calculation of the velocity field about a thin wing, including compressibility effects, is described in Sedov (1965: Chapter 2, pp 87-107). In general no simple expression for the slip velocity can be obtained. Here we consider two cases that do lead to relatively simple results. The first case is that of extremely low Mach numbers, such that the acoustic wavelength is long not only compared to the hydrodynamic length scale, $U_{\infty} / \omega$, but also compared to the airfoil chord, i.e., $k \ll 1$. In this situation the unsteady interaction of the acoustic wave with the airfoil can be analyzed using the classical unsteady airfoil theory for incompressible flow. The second case is that of acoustic wavelengths long compared to $U_{\infty} / \omega$ but short compared to the airfoil chord, i.e., $k \gg 1$. In this case acoustic diffraction theory can be used to analyze the interaction.

First consider the case $k \ll 1$, where incompressible, unsteady airfoil theory applies (see for example, Garrick, 1957). Since the airfoil semi-chord is the relevant length scale for the unsteady aerodynamic interaction, the airfoil thickness can be ignored (except in the region of the leading-edge, where (4.1) is applicable). Thus, the airfoil reduces to a zero-thickness flat plate, and the solution consists of two potential flow components, a non-ciculatory component, plus a component due to the vorticity shed from the sharp 
trailing edge. The shed vorticity is convected downstream by the mean flow and hence takes the form $\gamma(x, t)=\hat{\gamma} \mathrm{e}^{\mathrm{i}(a x-t)}$ where $a=\omega b / U_{e}$ is the aerodynamic reduced frequency. The non-dimensional complex potential for this unsteady flow is given by

$$
w=\frac{\mathrm{e}^{-\mathrm{i} t}}{2}\left(\mathrm{e}^{-\mathrm{i} \theta} \zeta+\frac{\mathrm{e}^{\mathrm{i} \theta}}{\zeta}\right)-\frac{\mathrm{i} \hat{\gamma} \mathrm{e}^{-\mathrm{i} t}}{2 \pi} \int_{2}^{\infty} \mathrm{e}^{\mathrm{i} a x_{0}} \ln \left(\frac{\zeta-\zeta_{0}}{\zeta-1 / \zeta_{0}}\right) \mathrm{d} x_{0},
$$

where $\zeta(z)=z-1+\sqrt{z(z-2)}$ and $\zeta_{0}=\zeta\left(x_{0}\right)$. Applying the unsteady Kutta condition then fixes the strength of the shed vorticity,

$$
\hat{\gamma}=\frac{2 \pi \sin \theta}{\int_{2}^{\infty} \mathrm{e}^{\mathrm{i} a x_{0}}\left(\frac{x_{0}}{x_{0}-2}\right)^{\frac{1}{2}} \mathrm{~d} x_{0}} .
$$

For matching with the local solution (4.1), the small- $x$ expansion of the velocity on the airfoil surface is required. We find

$$
u(x, 0 \pm) \sim\left\{ \pm \frac{\sin \theta}{2^{\frac{1}{2}}}(1+J(a)) x^{-\frac{1}{2}}+\cos \theta+O\left(x^{\frac{1}{2}}\right)\right\} \mathrm{e}^{-\mathrm{i} t} \quad \text { as } \quad x \rightarrow 0,
$$

where $J(a)$ represents the effect of the shed vorticity,

$$
J(a)=-\frac{\int_{2}^{\infty} \mathrm{e}^{\mathrm{i} a x_{0}}\left(\frac{x_{0}-2}{x_{0}}\right)^{\frac{1}{2}} \mathrm{~d} x_{0}}{\int_{2}^{\infty} \mathrm{e}^{\mathrm{i} a x_{0}}\left(\frac{x_{0}}{x_{0}-2}\right)^{\frac{1}{2}} \mathrm{~d} x_{0}}=O\left(\frac{1}{a}\right), \quad \text { as } \quad a \rightarrow \infty .
$$

Thus for the high reduced aerodynamic frequencies of interest in the present study, the effect of shed vorticity on the unsteady flow in the vicinity of the leading edge is smaller than the contribution due to the non-circulatory component, by a factor of $O(1 / a)$. Matching with the large $\xi$ limit of the local leading-edge solution (4.1) then gives

$$
\kappa_{s}=\cos \theta, \quad \kappa_{a}=a^{\frac{1}{2}} \sin \theta .
$$

The symmetric and anti-symmetric components of the local flow past the leading edge are seen to be in phase, owing to the incompressible nature of the unsteady interaction. Except for values of $\theta$ near 0 and $\pi$, the slip velocity near the leading edge is dominated by the anti-symmetric component of (4.1), which reaches a peak value $\left(b / r_{n}\right)^{1 / 2} \sin \theta$ at the nose of the airfoil. Note also that, in this limit, the flow about the nose (and hence the Receptivity Coefficient) is the same for an airfoil with sharp trailing edge as for a body with a rounded trailing edge.

Next consider the opposite limiting case, $k \gg 1$. The acoustic wavelength is then short compared to the airfoil chord, and the interaction of the acoustic wave with the leading edge can be analyzed by taking the airfoil chord to be semi-infinite. As in the first case, on the scale of the acoustic wavelength, the airfoil appears at leading order as a zero-thickness plate and the problem reduces to the classical Sommerfeld diffraction problem (Noble, 1958). The velocity potential for the unsteady flow is given by

$\phi(x, y)=\left\{-\frac{\mathrm{i}}{k} \mathrm{e}^{\mathrm{i} k(x \cos \theta+y \sin \theta)}-\sin \frac{1}{2} \theta \frac{\operatorname{sgn}(y)}{\pi(2 k)^{\frac{1}{2}}} \int_{-\infty}^{\infty} \frac{\exp \left(-\left(\lambda^{2}-k^{2}\right)^{\frac{1}{2}}|y|-\mathrm{i} \lambda x\right)}{(\lambda+k \cos \theta)(\lambda+k)^{\frac{1}{2}}} \mathrm{~d} \lambda\right\} \mathrm{e}^{-\mathrm{i} t}$,

which leads to a slip velocity

$$
u(x, 0 \pm)=\left\{\cos \theta \mathrm{e}^{\mathrm{i} k \cos \theta}(1 \mp \operatorname{erf} \Phi) \pm \sin \frac{1}{2} \theta \frac{2^{\frac{1}{2}} \mathrm{e}^{\frac{1}{4} \mathrm{i} \pi}}{(\pi k)^{\frac{1}{2}}} x^{-\frac{1}{2}}\right\} \mathrm{e}^{-\mathrm{i} t},
$$


where $\Phi=\mathrm{e}^{-\frac{1}{4} \mathrm{i} \pi}(2 k x)^{\frac{1}{2}} \sin \frac{1}{2} \theta$. Hence, as $x \rightarrow 0$,

$$
u(x, 0 \pm) \sim\left\{ \pm \sin \frac{1}{2} \theta \frac{2^{\frac{1}{2}} \mathrm{e}^{-\frac{1}{4} \mathrm{i} \pi}}{(\pi k)^{\frac{1}{2}}} x^{-\frac{1}{2}}+\cos \theta+O\left(x^{\frac{1}{2}}\right)\right\} \mathrm{e}^{-\mathrm{i} t} .
$$

Matching the slip velocity on the upper surface to the local solution in the vicinity of the leading edge, then gives

$$
\kappa_{s}=\cos \theta, \quad \kappa_{a}=\frac{2 \mathrm{e}^{-\frac{1}{4} \mathrm{i} \pi}}{\left(\pi M_{e}\right)^{\frac{1}{2}}} \sin \left(\frac{1}{2} \theta\right),
$$

where $M_{e}=U_{e} / c$ is the Mach number of the mean flow, which has been assumed small. The anti-symmetric component of the local flow past the leading edge lags the incident field (and the symmetric component) by a phase angle $\pi / 4$, owing to the influence of compressibility. The anti-symmetric component again dominates the symmetric component, except for values of $\theta$ near zero. However, the dependence on the incidence angle $\theta$ is fundamentally different than for the case of small $k$. The anti-symmetric component of the slip velocity has a peak value $2 \mathrm{e}^{\mathrm{i} \pi / 4}\left(c / \pi \omega r_{n}\right)^{\frac{1}{2}} \sin \frac{1}{2} \theta$ at the nose, a result that depends only on the compressibility of the fluid and not on the airfoil semi-chord $b$.

In the next section, the symmetric and anti-symmetric components, $C_{s}(S)$ and $C_{a}(S)$, of the Receptivity Coefficient are calculated, and sample results are presented illustrating the dependence of the Receptivity Coefficient $C_{1}(S)$ on the incidence angle $\theta$ of the acoustic wave in the limits $k \ll 1$ and $k \gg 1$.

\section{Numerical Results}

In $\S 3$, we utilised asymptotic methods to obtain the non-Blasius generalisations of the Lam-Rott asymptotic eigenfunctions, the first of which is the precursor of the TollmienSchlichting wave. The asymptotic analysis determines the form of these eigenfunctions, but not their coefficients $C_{i}$. It appears that the Receptivity Coefficient $C_{1}$ for a particular free-stream disturbance can be determined only by numerically solving the LUBLE over the full range of $\xi$ and examining the behavior for large $\xi$.

In contrast to the flat-plate studies of previous authors, where the mean flow is known throughout, in the present study the mean flow, governed by (3.5), must be computed as well as the unsteady disturbance, governed by (3.7). The initial conditions at $\xi=0$, which correspond to the steady Hiemenz flow and its quasi-steady linear perturbation, take the form of ODEs in $\eta$, which were solved using a fourth-order Runge-Kutta method. The solution was then obtained by marching downstream using a Keller Box scheme (Keller \& Cebeci, 1970) for both the mean flow, given by a nonlinear PDE (3.5), and the linearised disturbance equations for symmetric and anti-symmetric components, (4.4). From the asymptotic form of the eigensolutions, it is clear that the growth or decay of the disturbance is very sensitive to the mean flow. For this reason, the departure of the mean flow away from the Blasius solution was calculated rather than the mean flow directly. The value of the unknown coefficient $B_{1}$ in (3.9) can then be extracted from the wall shear. From the asymptotic expansion for the mean flow close to the wall (3.11),

$$
\left(\frac{\bar{\xi}^{2}[\phi-F]_{\eta \eta}}{\left(\ln \bar{\xi}^{2}\right) F_{\eta \eta}}\right)_{\eta=0} \sim A_{1}+\frac{B_{1}}{\ln \bar{\xi}^{2}}+O\left(\bar{\xi}^{-1.774}(\ln \bar{\xi})^{-1}\right) .
$$

From figure 3 , where the above quantity is plotted for real $\bar{\xi}$, comparison of the numerical result with this asymptotic form gives $B_{1} \approx 2.08$. This is somewhat higher than the value suggested by Van Dyke (1964b), who estimated $B_{1} \approx 1.6-1.9$ based on coarse numerical 


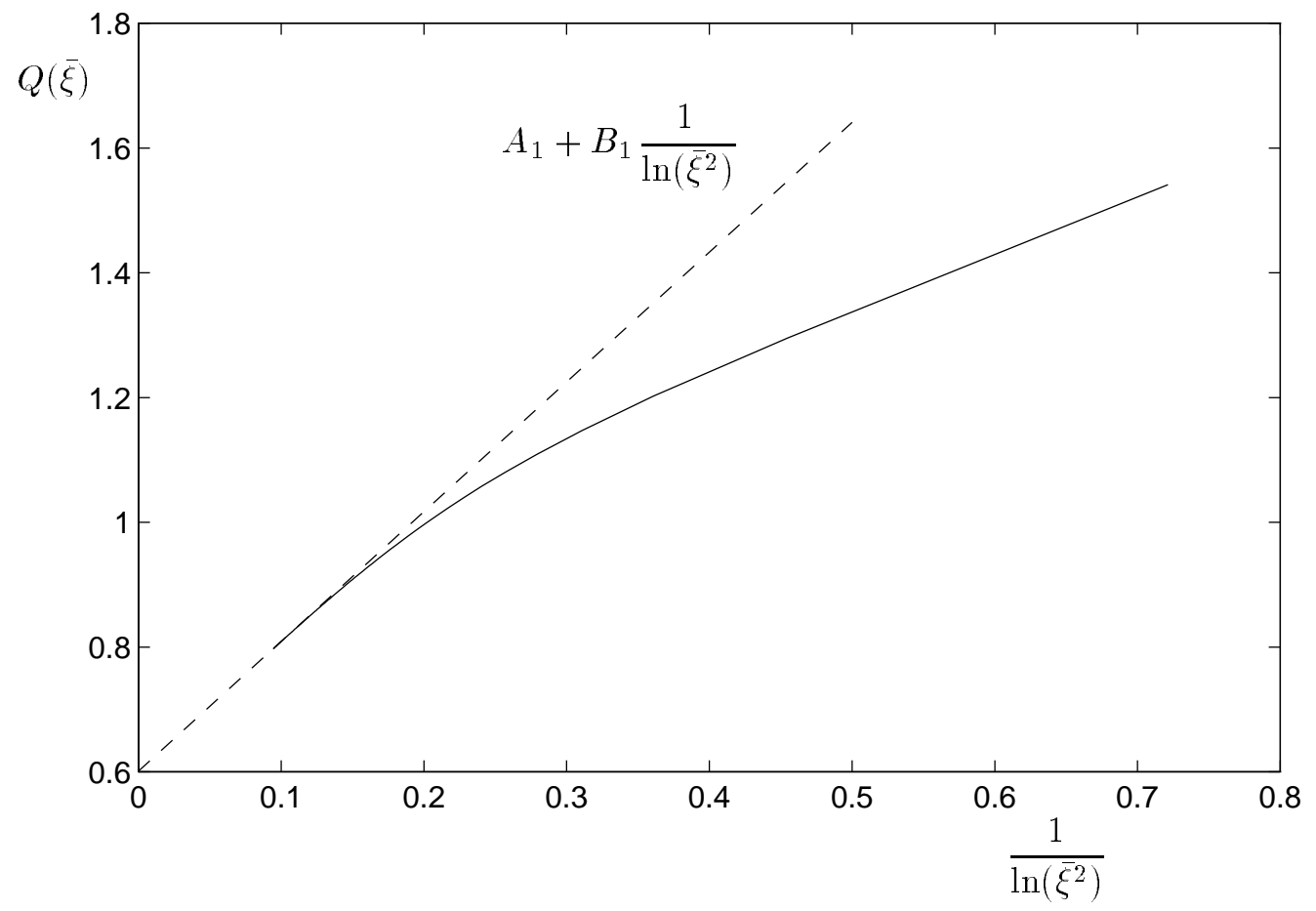

Figure 3. Plot of $Q(\bar{\xi})=\left(\ln \bar{\xi}^{2}\right)^{-1} \bar{\xi}^{2}\left(\left[\phi^{\prime \prime}-F^{\prime \prime}\right] / F^{\prime \prime}\right)_{\eta=0}$ against $\left(\ln \bar{\xi}^{2}\right)^{-1}$. Comparison with the large $\bar{\xi}$ asymptotic form, (3.9) yields $B_{1} \approx 2.08$.

results and on numerical patching of the asymptotic series valid near the nose to the series valid far downstream. The computations undertaken in the present work extend to much higher values of $\bar{\xi}$, allowing much more accurate extrapolation to fix $B_{1}$. In addition, the magnitude of the next term in the asymptotic series can be estimated from figure 3 . By considering the magnitude of the correction term at $\bar{\xi}^{2}=30$, the coefficient of the next term in expansion (5.1) is estimated to be approximately 10, which probably explains the under-estimation of Van Dyke.

We turn now to the solution for the disturbance. Since the real part of $T(\xi)$ is negative, the first Lam-Rott eigensolution becomes exponentially small far downstream, compared to the Stokes wave. Moreover, since the eigenvalues are inversely ordered, the first eigensolution is also exponentially small compared to all the other eigensolutions. Thus, it is very difficult to extract the coefficient of the eigensolution by direct numerical solution of the LUBLE. A way round this difficulty is to move the integration off the real line into the complex $\xi$-plane in such a way that the eigensolution will grow exponentially (Goldstein et al, 1983). This will occur if the streamwise variable $\xi$ is chosen such that $-5 \pi / 12<\arg \xi<-\pi / 12$. The inverse ordering of the eigensolutions is also rectified by this process, the first eigenfunction becoming exponentially dominant for $|\xi| \gg 1$. 'Peeling off' the exponentially growing part of the first eigensolution, that is solving for $f=\psi \mathrm{e}^{-T_{1}^{(0)}}$ rather than $\psi$, allows the numerical solution to be continued much further downstream while retaining accuracy (Heinrich \& Kerschen, 1989).

There are essentially two ways of obtaining the Receptivity Coefficient $C_{1}$, one based on the wall shear and the other based on the oscillating boundary-layer thickness. The 
latter method was used by Goldstein et al (1983) and Heinrich \& Kerschen (1989) for the flat-plate analysis. In the present work the two methods gave virtually identical results, small differences only arising due to the difference in large- $\xi$ extrapolation procedure in the two cases. Using the wall shear as the basis for comparison, we define

$$
\mathcal{C}(S, \xi)=\frac{f_{\mathrm{Num}}^{\prime \prime}(\eta=0)}{f_{0}^{\prime \prime}}
$$

where $f_{0}^{\prime \prime}=0.4356(1+\mathrm{i})\left(\xi^{2} / 2\right)^{\tau_{1}+1}$. Since the argument of $\xi$ was chosen so that the first eigensolution dominates other components of the solution, $f \sim C_{1} \psi_{1} \mathrm{e}^{-T_{1}^{(0)}(\xi)}$, and hence

$$
\mathcal{C} \sim C_{1}\left[1+b_{1} S^{1.887} \frac{1}{\xi^{0.774}}+b_{2} S^{2} \frac{\ln ^{2}\left(\xi^{2} / S\right)}{\xi}+b_{3} S^{2} \frac{\ln \left(\xi^{2} / S\right)}{\xi}+b_{4} S^{2} \frac{1}{\xi}+\ldots\right],
$$

as $\xi \rightarrow \infty$. Thus in principle, $C_{1}$ can be extrapolated by plotting the numerically obtained value of $\mathcal{C}(S, \xi)$ against $\xi^{-0.774}$. However, this method has serious limitations as far as obtaining highly accurate estimates for $C_{1}$. Until $\xi$ is extremely large, the sizes of the next three terms in the expansion are very close to that of the leading order correction, with the relative magnitudes depending also on $S$. In addition, the analysis of the base flow suggests that $b_{1}$ is quite large, so for larger values of $S$ the exponential term cannot be expanded out until $\xi$ is very large. Due to these difficulties, the Receptivity Coefficient for non-zero $S$ cannot be calculated to the same accuracy as for the flat plate, the inaccuracy increasing as $S$ increases. In our calculations, we usually chose $\arg \xi=-\pi / 4$ which ensures that the rate of growth of the first Lam-Rott eigensolution is maximised. In addition, for this angle any error in the numerically determined value of $B_{1}$ enters only the phase of $C_{1}$ and not the magnitude. For some values of $S$, the solution for $\arg \xi=-\pi / 3$ was also obtained and the extrapolated value of $C_{1}$ was virtually identical, further verifying the numerical work as well as the functional form of the eigensolution. The solution was integrated up to $|\xi|=15$, except for the larger values of $S$ where it was continued up to $|\xi|=20$ in an attempt to improve the extrapolation. Computation to larger values of $\xi$ becomes progressively more expensive due to the presence of the viscous wall layer, the relative thickness of which decreases downstream.

In figure 4, the numerically obtained value of the Receptivity Coefficient for acoustic waves propagating parallel to the airfoil chord, $C_{s}$, is plotted for several values of $S$. In figure $4 a$ it is seen that the magnitude of the Receptivity Coefficient increases slightly for very small $S$, but decreases rapidly as the nose radius increases further. For $S=0.3$, the receptivity is reduced to approximately $15 \%$ of that for a flat plate. The decay of $\left|C_{s}\right|$ appears to be exponential in $S$, though this has not been verified analytically. For $S \leq 0.1$, the results for $\left|C_{s}\right|$ are estimated as accurate to $2 \%$, while the accuracy for $S=0.3$ is only about $10 \%$, due to the difficulties discussed above. However, the large relative uncertainty for the latter case is of little practical importance, since the receptivity is so low by this stage. The small increase in the parallel-wave receptivity seen for very small values of $S$ is in agreement with small- $S$ asymptotic theory which will be reported elsewhere (Hammerton \& Kerschen 1996a). Figure $4 b$ shows the change in the phase of the Receptivity Coefficient as $S$ increases. The increase in $\arg \left(C_{s}\right)$ corresponds to a lag in phase of the instability wave.

As we have already noted, computation must be carried out to large values of $\xi$ for accurate determination of the Receptivity Coefficient, since the eigensolution tends to its asymptotic limit slowly. However, the ratio of Receptivity Coefficients for different free-stream disturbances can be evaluated accurately at only moderately large values of $\xi$, since the same asymptotic behaviour of the eigensolution arises whatever the form 
Figure $4 a$

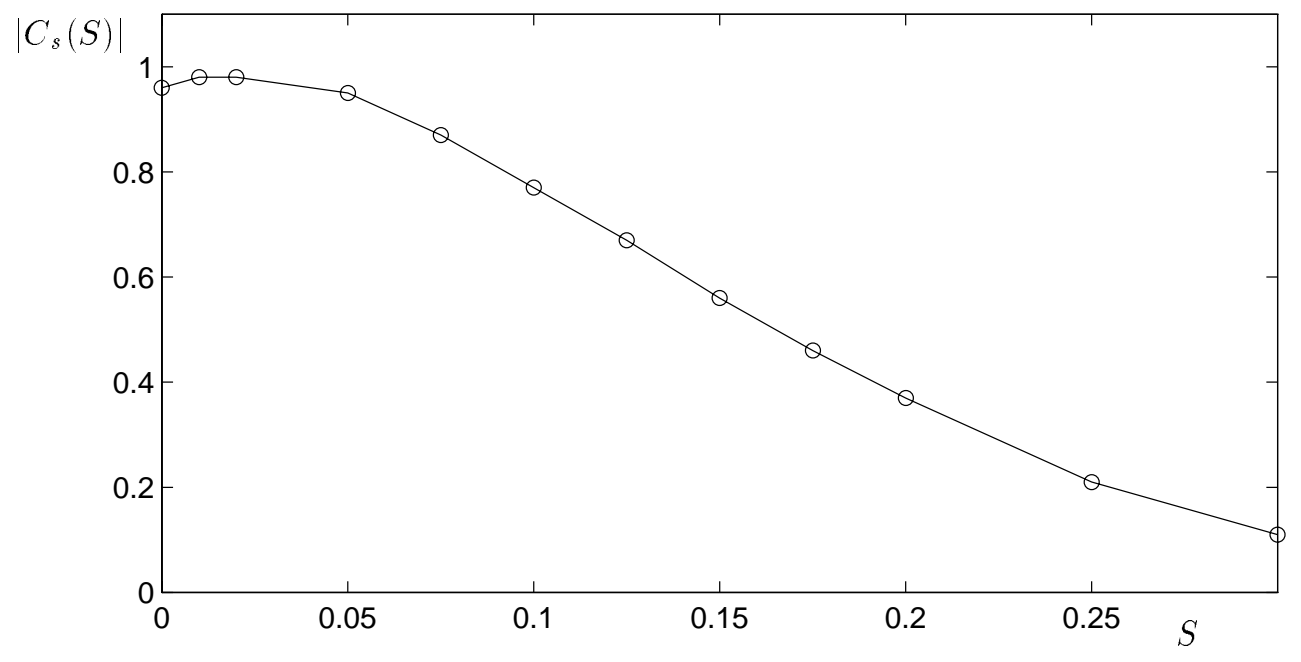

Figure $4 \mathrm{~b}$

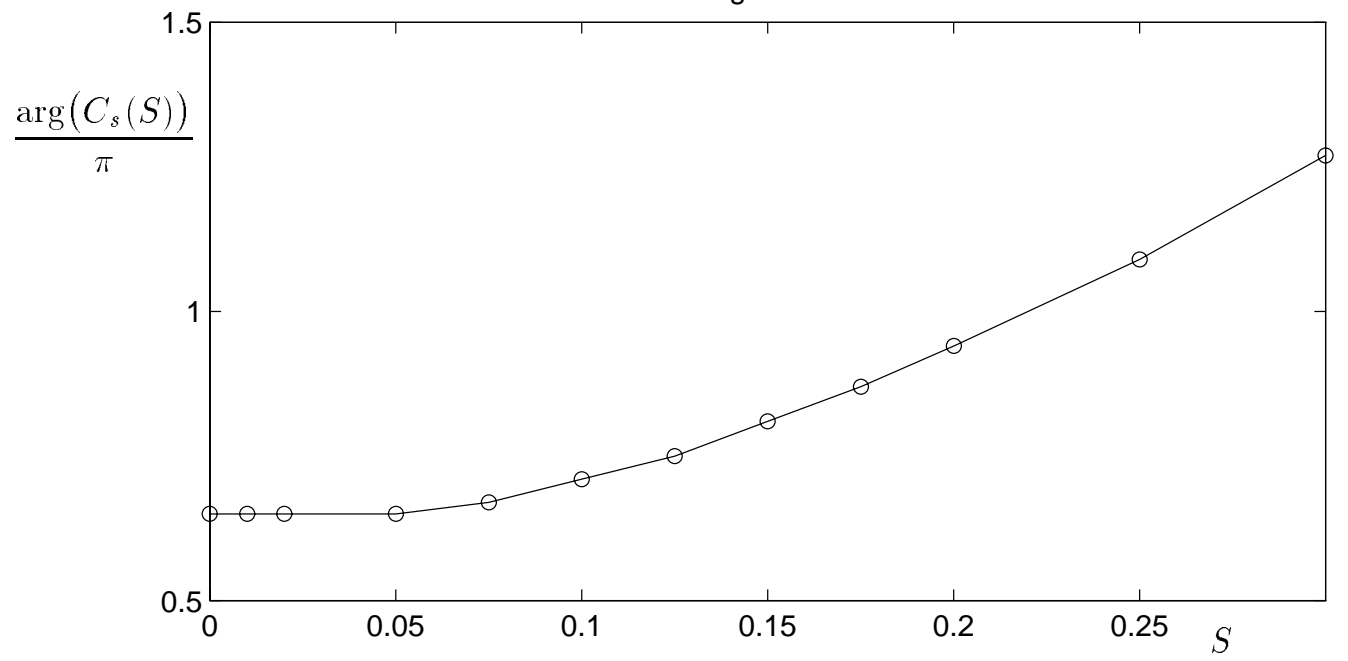

FiguRE 4. Variation of the Receptivity Coefficient as a function of Strouhal number, $S$, for an acoustic wave in the free stream propagating parallel to the body axis: (a) the amplitude of the Receptivity Coefficient $\left|C_{s}(S)\right|$; (b) the phase of the Receptivity Coefficient $\arg \left(C_{s}\right) / \pi$.

of the free-stream disturbance. In the present paper, we consider only the effect of acoustic waves at oblique angles. In figure 5, the receptivity due to the symmetric and anti-symmetric components of the free-stream disturbance is compared for different nose radii. We define $\Lambda=C_{a} / C_{s}$, where $C_{a}$ is the Receptivity Coefficient related to the antisymmetric component of (4.1). In figure $5 a,|\Lambda|$ is plotted as a function of $S$. We see that $\left|C_{a}\right|$ is larger than $\left|C_{s}\right|$ by approximately a factor of six in the flat-plate limit $S \rightarrow 0$, where the anti-symmetric component of the slip velocity has a square-root singularity at the leading edge. The value of $|\Lambda|$ decreases rapidly as a finite nose radius is introduced, relieving the singularity at the leading edge. Thus, the behaviour of $C_{a}$ for very small $S$ 
Figure $5 \mathrm{a}$

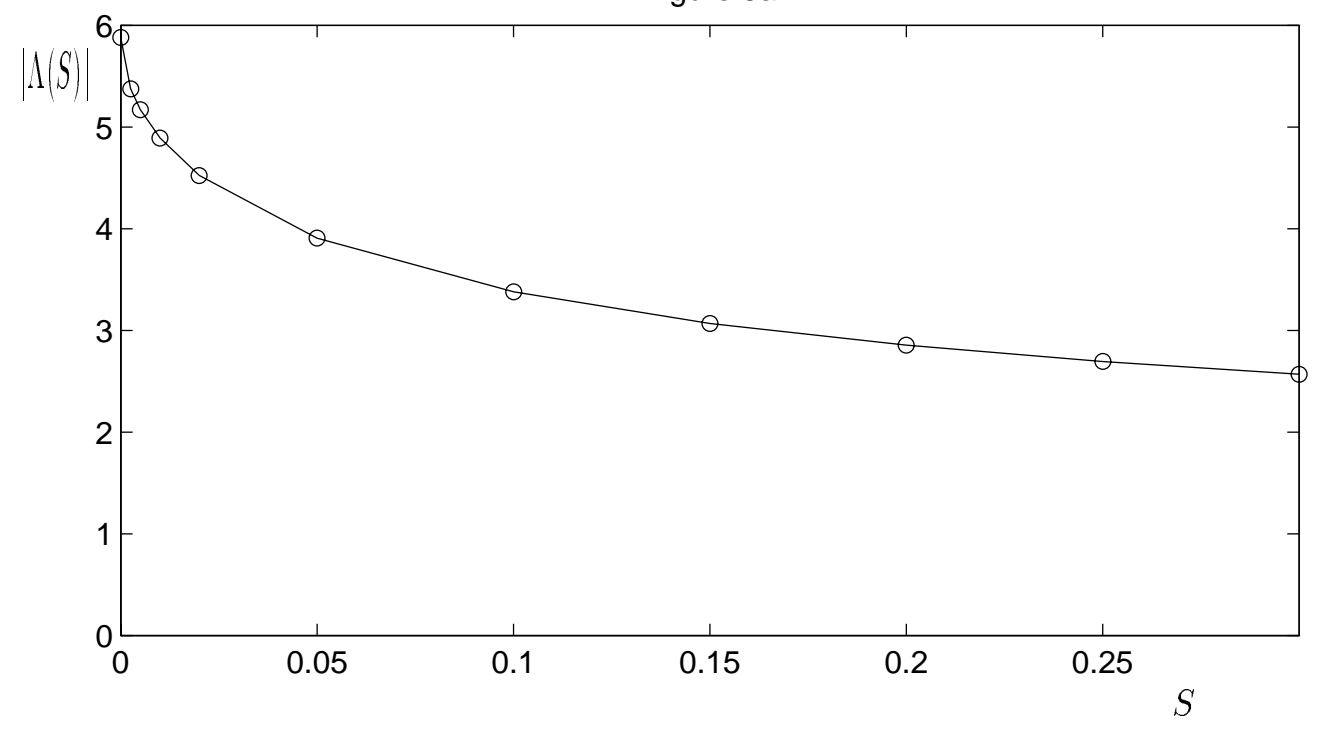

Figure $5 b$

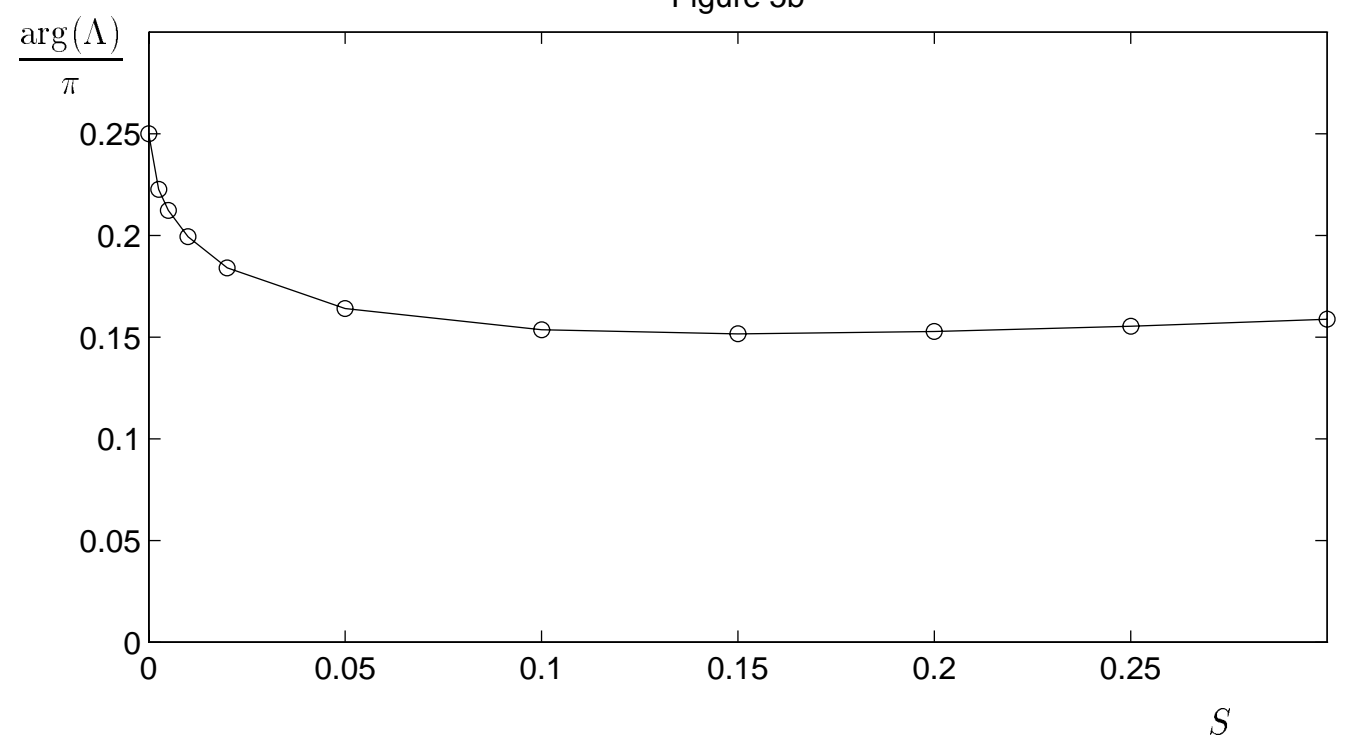

FiguRE 5. Comparison of the relative receptivity due to symmetric and asymmetric components of an oblique acoustic wave, $\Lambda=C_{a} / C_{s}$ : (a) the magnitude of the relative receptivity, $|\Lambda|$; (b) the phase, $\arg (\Lambda)$.

is quite different from that of $C_{s}$, where a small rise in magnitude was seen. The sharp change in $\Lambda$ for small $S$ suggests the appearance of singular behaviour in the small- $S$ expansion, in contrast to the behaviour seen in figure 4 for the parallel-wave case. Beyond $S=0.05$ the decline in $|\Lambda|$ becomes more gradual, the value of $|\Lambda|$ remaining above 2.5 right out to $S=0.3$. For all values of $S$ examined, the anti-symmetric component of the 


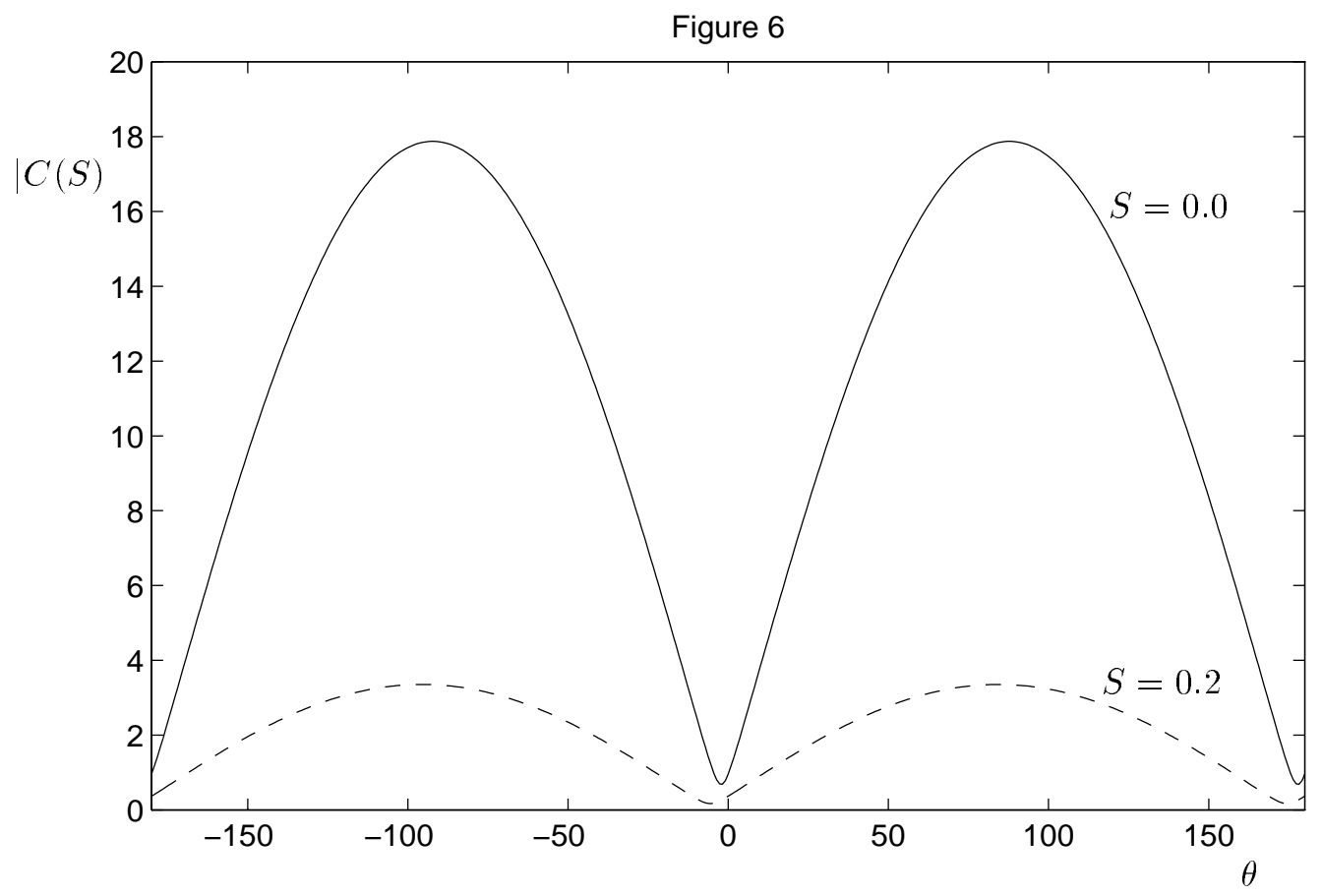

Figure 6. Variation of $|C|$ with acoustic incidence angle $\theta$, for $k \ll 1$ and non-dimensional airfoil chord length $a=10$. The solid line is for $S=0.0$, the dotted line $S=0.2$.

slip velocity was found to be more effective than the symmetric component in generating a Tollmien-Schlichting wave.

The phase difference between the $C_{a}$ and $C_{s}$ is plotted in figure $5 b$. It is interesting to note that as $S \rightarrow 0, \arg \Lambda \rightarrow \pi / 4$. For reasons given above, $\Lambda$ can be calculated very accurately, and this result is true to an accuracy better than $0.1 \%$. However, the fundamental reason for this result is not clear. $\operatorname{Arg}(\Lambda)$ initially drops rapidly as a finite nose radius is introduced, then remains roughly constant from $S=0.05$ out to $S=0.3$. The positive values of $\arg (\Lambda)$ correspond to a phase lag for the contribution from the anti-symmetric component of slip velocity, relative to the contribution from the symmetric component. This phase lag may be related to the fact that the anti-symmetric component of the slip velocity takes on its largest values near the nose, while the symmetric component takes on its largest value farther downstream. The concentration of the anti-symmetric component near the leading edge is particularly pronounced in the limit $S \rightarrow 0$, where $\arg (\Lambda)$ takes on its largest value, the slip velocity being singular at $\xi=0$ in this case.

The variation of the total Receptivity Coefficient $C_{1}$ with acoustic wave incidence angle is illustrated for the case $k \ll 1$ in figure 6 . A representative aerodynamic reduced frequency, $a=10$, has been chosen, and results are plotted for two nose radii, $S=0$ and 0.2 . The receptivity for the flat-plate case $(S=0)$ is dominated by the contribution from the anti-symmetric component, causing the shape of the plot for $\left|C_{1}\right|$ to be quite close to $\sin \theta$, except in the vicinity of $\theta=0^{\circ}$ and $180^{\circ}$ where the level is determined by the symmetric component. The case $a=10, S=0.2$ corresponds to a typical airfoil design. The overall receptivity level for $S=0.2$ is smaller than for the flat plate, due to decreases in both $\left|C_{s}\right|$ and $\left|C_{a}\right|$. Since the finite nose radius causes a larger decrease in 


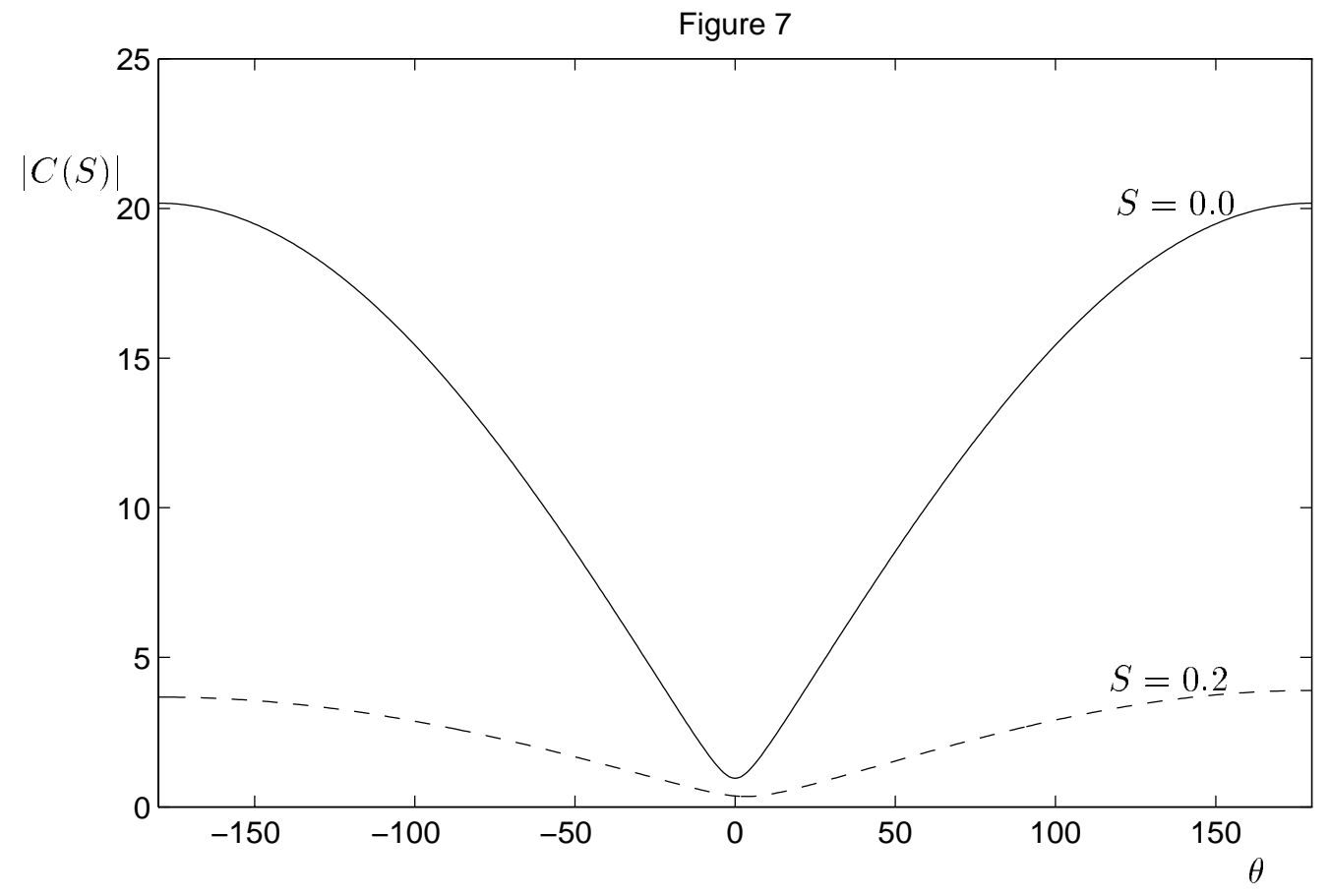

Figure 7. Variation of $|C|$ with acoustic incidence angle $\theta$, for $k \gg 1$ and $M=0.1$, and the same values of $S$ plotted in figure 6 .

$C_{s}$ than $C_{a}$, the influence of the symmetric component of the free-stream disturbance is somewhat larger in this case. The peak receptivity for $S=0.2$ is roughly one-fifth that for the flat-plate.

The variation of the total Receptivity Coefficient $C_{1}$ with incidence angle is illustrated for the case $k \gg 1$ in figure 7 . A representative Mach number $M_{e}=0.1$, has been chosen. The dependence of the receptivity level on acoustic wave incidence angle is very different from the case $k \ll 1$ illustrated in figure 6 . The flat-plate result is again dominated by the contribution from the anti-symmetric component of the free-stream disturbance, but the plot-shape resembles $\sin \frac{1}{2} \theta$, except near $\theta=0^{0}$ where the symmetric component determines the level. As before, for $S=0.2$ the overall receptivity level is decreased, but the relative contribution from the symmetric component of the disturbance is somewhat larger.

\section{Conclusion}

Boundary layer transition is influenced both by the stability properties of the boundary layer, and by the characteristics of the free-stream disturbances and the receptivity of the boundary layer to these disturbances. The present work has examined the influence on leading-edge receptivity of the nose radius of an uncambered airfoil symmetric mean flow, in the low Mach number limit.

Leading-edge receptivity involves a gradual evolution of the boundary-layer disturbances with downstream distance, eventually leading to a growing Tollmien-Schlichting wave. The Receptivity Coefficient is essentially the amplitude of the asymptotic eigenfunction which is the precursor of the Tollmien-Schlichting wave. It is the Receptivity 
Coefficient which contains all the relevant information about the free-stream disturbance. The decay rate of the asymptotic eigenfunction, like that of the Tollmien-Schlichting wave, is influenced only by the mean flow. One of the most important properties is the pressure gradient parameter, $\beta$. For symmetric flow past a parabola,

$$
\beta=\frac{S}{\xi^{2}+S},
$$

where $S$ is the Strouhal number, $S=\omega r_{n} / U_{e}$. Thus, the pressure gradient is everywhere favorable, decreasing monotonically from its maximum value at the stagnation point and approaching zero far downstream. Well downstream of the nose, the favorable pressure gradient has a stabilising influence on the boundary layer, leading to increased damping of the Tollmien-Schlichting waves and of the precursor disturbances. In addition, the stronger pressure gradient close to the nose has an effect on the Receptivity Coefficient. As the nose radius of the body is increased (i.e. $S$ increased), the strongly favorable pressure gradient near the nose extends over a larger number of disturbance wavelengths, and this additional stabilising influence is likely to result in a decrease in the Receptivity Coefficient. Numerical calculations show that the symmetric component $C_{s}$ of the Receptivity Coefficient decreases rapidly with increasing $S$, dropping to approximately $12 \%$ of the flat-plate value when $S=0.3$. The anti-symmetric component $C_{a}$ decreases even more rapidly, dropping to $5 \%$ of flat-plate value when $S=0.3$. The total Receptivity Coefficient $C_{1}$ is much larger for oblique acoustic waves than for parallel acoustic waves, since the coefficient $\kappa_{a}(\theta)$ that multiplies the anti-symmetric component $C_{a}$ is large compared to the coefficient $\kappa_{s}(\theta)$ that multiplies the anti-symmetric component $C_{s}$.

While the detailed asymptotic analysis depends on the exact geometry of the leading edge, the rapid decrease in receptivity with increasing nose radius should also be valid for other streamlined bodies. However, symmetric mean flows past leading edges less streamlined often involve regions of adverse as well as favorable pressure gradient close to the nose. For such bodies, it is possible that an increase in leading edge thickness could lead to an increase in the Receptivity Coefficient. For an airfoil at an angle of attack, both favorable and adverse pressure gradients exist in the neighborhood of the nose. Analysis of the receptivity in this case will be presented in a future paper (Hammerton \& Kerschen 1996b).

Comparing the asymptotic analysis presented here with numerical and experimental results is difficult. Numerical work by Murdock (1981) for a parabola in a flow with a parallel acoustic wave also showed a decrease in receptivity as the nose radius was increased. The computations of Lin et al. (1992) were for parallel acoustic waves incident on half-ellipse leading edges connected to a flat plate and for super-ellipse leading edges (which avoid the discontinuity in curvature), geometries chosen to match the experiments of Saric et al. (1994). For both these geometries there are regions of adverse pressure gradient near the leading edge, as well as the possibility of additional localised receptivity mechanisms (Goldstein, 1985); hence no direct comparisons can be made with the present results. In addition, the receptivity level 'seen' in experiments and full NavierStokes calculations is a combination of the receptivity process described in the current paper, and the stability characteristics farther downstream. For the flat-plate, Goldstein (1983) provided the asymptotic analysis of the triple-deck region and thus (in theory) could calculate the disturbance amplitude at the lower branch. Reproducing such an analysis for a general airfoil surface would provide little additional physical insight concerning the receptivity process. By restricting attention to the region in which forcing by the free-stream disturbance occurs, the process of receptivity is isolated from the instability phenomena that occut farther downstream. For a global picture of the transition 
process, the current analysis provides an upstream boundary condition for linear stability analyses.

This work was supported by NASA Langley Research Center under grant NAG-1-1135 and Air Force Office of Scientific Research under grant 90-0065.

\section{Appendix A. Evaluation of Integrals involving Airy Functions}

Here we obtain alternative expressions for the integrals $J_{i}$, defined by

$$
J_{i}=\int_{z_{0}}^{\infty}\left[z-z_{0}\right]^{i} \mathrm{Ai}^{2}(z) \mathrm{d} z
$$

where $\operatorname{Ai}^{\prime}\left(z_{0}\right)=0$. Setting $w=\operatorname{Ai}^{2}(z)$, this satisfies

$$
w^{\prime \prime \prime}-4 z w^{\prime}-2 w=0
$$

(Abramowitz \& Stegun (1965); equation 10.4.57), and hence

$$
\int^{z} w\left(z^{\prime}\right) \mathrm{d} z^{\prime}=-\frac{1}{2}\left(w^{\prime \prime}-4 z w\right) .
$$

Thus $J_{0}\left(z_{0}\right)$ follows, noting that $w^{\prime \prime}\left(z_{0}\right)=2 z_{0} \operatorname{Ai}^{2}\left(z_{0}\right)$. The other integrals $J_{i}$ are then obtained by successive integration by parts,

$$
\begin{aligned}
J_{i} & =\frac{i}{2} \int_{z_{0}}^{\infty}\left(z-z_{0}\right)^{i-1}\left(w^{\prime \prime}-4 z w\right) \mathrm{d} z \\
& =-2 i J_{i}-2 i z_{0} J_{i-1}+\frac{i}{2} \int_{z_{0}}^{\infty}\left(z-z_{0}\right)^{i-1} w^{\prime \prime} \mathrm{d} z .
\end{aligned}
$$

Hence,

$$
\left.\begin{array}{rl}
J_{0} & =-z_{0} \mathrm{Ai}^{2}\left(z_{0}\right), \\
J_{1} & =-\frac{2}{3} z_{0} J_{0}, \\
J_{2} & =\frac{1}{5}\left(-4 z_{0} J_{1}+\operatorname{Ai}^{2}\left(z_{0}\right)\right), \\
J_{i} & =-\frac{2 i}{1+2 i} z_{0} J_{i-1}+\frac{i(i-1)(i-2)}{2(1+2 i)} J_{i-3}, \quad i \geq 3 .
\end{array}\right\}
$$

\section{Appendix B. Matching of Asymptotic Eigenfunctions to Tollmien-Schlichting Modes}

As the wavelength of the asymptotic eigenfunctions of the Linearised Unsteady Boundary Layer Equations progressively shortens with distance downstream, an outer inviscid layer begins to interact with the wall viscous layer. This occurs when $\xi=O\left(\epsilon^{-1}\right)$. Writing $\hat{\xi}=\epsilon \xi$, we anticipate the rapid variation of $\psi^{\prime}$ by writing

$$
\psi^{\prime}=G(\eta, \hat{\xi}) \exp \left(\mathrm{i} \int k d \hat{\xi} / \epsilon^{3}\right)
$$

where the scaling is dictated by the need to match back to (3.17) as $\hat{\xi} \rightarrow 0$. As in the LUBLE regime, the exponential streamwise variation $k(\hat{\xi})$ is determined as an eigenvalue from the matching between the different layers. In the Orr-Sommerfeld region, the main deck $(\eta=O(1))$ is matched to an outer irrotational layer $\left(\eta=O\left(\epsilon^{-1}\right)\right)$ and to the inner viscous layer $(\eta=O(\epsilon))$. When $\xi=O\left(\epsilon^{-1}\right)$, the classic triple deck scalings arise and 
the effect of the outer layer enters the expression for $k$ at leading order. In the present investigation, $k(\hat{\xi})$ was determined using direct matching of the stream function between the decks, rather than by trying to modify standard Orr-Sommerfeld results to include wall curvature and mean pressure gradients effects.

Details of the matching are not included here, but an implicit expression for $k(\hat{\xi}, \epsilon)$ is eventually obtained,

$$
\widehat{Q}\left(z_{0}\right)=\gamma^{-3}\left(1-\frac{2 S}{\hat{\xi}^{2}} \epsilon^{2}\right) h\left(z_{0}, \epsilon, \hat{\xi}\right)
$$

where

$$
\widehat{Q}\left(z_{0}\right) \equiv \frac{\mathrm{e}^{\frac{1}{2} \mathrm{i} \pi} z_{0}^{2} \mathrm{Ai}^{\prime}\left(z_{0}\right)}{\int_{\infty}^{z_{0}} \operatorname{Ai}(\tilde{z}) \mathrm{d} \tilde{z}}, \quad z_{0}=\left(\frac{-\mathrm{i} \widehat{H}^{6}}{{U_{0}^{\prime}}^{2} \gamma^{2} \hat{\xi}^{2} k^{2}}\right)^{\frac{1}{3}}, \quad \gamma=1+A \frac{\ln \xi^{2} / S}{\xi^{2} / S}+B \frac{1}{\xi^{2} / S}
$$

and

$$
h\left(z_{0}, \epsilon, \hat{\xi}\right) \equiv \tilde{\xi}^{3}+\mathrm{e}^{\mathrm{i} \pi / 4} \tilde{\xi}^{2} z_{0}^{3 / 2}\left(2-\frac{\widehat{J_{1}} \tilde{\xi}^{3}}{\mathrm{i} z_{0}^{3}}\right) \epsilon+\tilde{\xi} z_{0}^{3} \mathrm{i}\left(1+\frac{2 \widehat{J}_{2} \tilde{\xi}^{3}}{\mathrm{i} z_{0}^{3}}-\frac{\widehat{J}_{3} \tilde{\xi}^{6}}{z_{0}^{6}}\right) \epsilon^{2}+O\left(\epsilon^{3} \ln \epsilon\right),
$$

Here $\tilde{\xi}=\hat{\xi} / U_{0}^{\prime}$ and $\widehat{J}_{i}$ are numerical constants involving integrals of the mean flow through the boundary layer, given by Goldstein (1983: equations 4.44-4.45). Solving (B 2) for $z_{0}(\hat{\xi}, \epsilon)$ gives

$$
z_{0}=\bar{z}_{0}\left(1+\frac{\tilde{\xi}^{3} \Gamma}{\bar{z}_{0} \widehat{Q}^{\prime}\left(\bar{z}_{0}\right)}\right)
$$

where $\bar{z}_{0} \sim z_{00}+\epsilon z_{01}+\epsilon^{2} z_{02}+O\left(\epsilon^{3} \ln \epsilon\right)$ is the flat-plate result, and

$$
\Gamma=-3 A \frac{\ln \xi^{2} / S}{\xi^{2} / S}-(3 B+2) \frac{1}{\xi^{2} / S}=O\left(\epsilon^{2} \ln \epsilon\right) .
$$

Here we have written $\gamma$ and $\Gamma$ in terms of $\xi$ rather than $\hat{\xi}$ for conciseness. Thus we finally obtain

$$
k=\bar{k}\left(1-A \frac{\ln \xi^{2} / S}{\xi^{2} / S}+\left(\frac{3}{2}-B\right) \frac{1}{\xi^{2} / S}\right)\left(1-\frac{3 \tilde{\xi}^{3} \Gamma}{2 \bar{z}_{0} \widehat{Q}^{\prime}\left(\bar{z}_{0}\right)}\right)
$$

where $\bar{k}=\mathrm{e}^{3 \mathrm{i} \pi / 4} \hat{\xi}^{2} \bar{z}_{0}^{-3 / 2} / U_{0}^{\prime}$ is the flat plate result.

The leading order term in the small- $\epsilon$ expansion of $\bar{z}_{0}$ is given implicitly by

$$
\widehat{Q}\left(z_{00}\right)=\tilde{\xi}^{3}
$$

which has a set of roots $z_{00}^{(i)}(\hat{\xi})$. As $\hat{\xi} \rightarrow 0, z_{00}^{(i)} \rightarrow-\rho_{i}$, where $\rho_{i}$ is defined in $\S 3$, namely the $i$-th root of $\operatorname{Ai}^{\prime}(-\rho)=0$. The set of roots $k^{(i)}(\hat{\xi})$ then follows from (B 5). As $\hat{\xi} \rightarrow 0$,

$$
k^{(i)} \sim \epsilon^{2} \frac{\mathrm{e}^{\frac{1}{4} \mathrm{i} \pi} \xi^{2}}{U_{0}^{\prime} \rho_{i}^{3 / 2}}\left(1-A \frac{\ln \xi^{2} / S}{\xi^{2} / S}+\left(\frac{3}{2}-B\right) \frac{1}{\xi^{2} / S}\right)
$$

and hence

$$
\frac{\mathrm{i}}{\epsilon^{3}} \int_{0}^{\hat{\xi}} k^{(i)} d \hat{\xi} \sim-\frac{\mathrm{e}^{-\frac{1}{4} \mathrm{i} \pi} \xi^{3}}{U_{0}^{\prime} \rho_{i}^{\frac{3}{2}}}\left(\frac{1}{3}-A_{1} S \frac{\ln \left(\xi^{2} / S\right)}{\xi^{2}}+\left(2 A_{1}+3 / 2-B_{1}\right) \frac{S}{\xi^{2}}\right),
$$

which matches to the exponential variation of the eigenfunctions of the LUBLE region (3.17). Numerical solution of $\widehat{Q}\left(z_{00}\right)=\tilde{\xi}^{3}$ shows that as $\tilde{\xi}$ increases, the imaginary part of the leading order term in $k^{(i)}$ stays positive for $i=2,3, \ldots$, corresponding to damped 
modes, but that $\operatorname{Im}\left(k^{(1)}\right)<0$ for $\tilde{\xi}>3.03$, which corresponds to a growing wave. Thus we have demonstrated that the first LUBLE eigenfunction does indeed match on to the unstable Tollmien-Schlichting mode. Moreover, (B 5) can be used to calculate the change in the position of the neutral stabilty point compared to the flat plate case.

\section{REFERENCES}

Abramowitz, M. \& Stegun, I. A. 1965 Handbook of Mathematical Functions. Dover.

Ackerberg, R.C. \& Phillips, J.H. 1972 The unsteady laminar boundary layer on a semiinfinite flat plate due to small fluctuations in the magnitude of the free-stream velocity. $J$. Fluid Mech. 51, 137-157.

Brown, S.N. \& Stewartson, K. 1973 On the propagation of disturbances in a laminar boundary layer. Proc. Camb. Phil. Soc. 73, 493-514.

GARRICK, I.E. 1957 Nonsteady wing characteristics. In Aerodynamic components of aircraft at high speeds (eds. A.F. Donovan \& H.R. Lawrence) pp. 658-794. Princeton University Press.

Goldstein, M.E. 1983 The evolution of Tollmien-Schlichting waves near a leading edge. $J$. Fluid Mech. 127, 59-81.

Goldstein, M.E. 1985 Scattering of acoustic waves into Tollmien-Schlichting waves by small streamwise variations in surface geometry. J. Fluid Mech. 154, 509-529.

Goldstein, M.E. \& Hultgren, L.S. 1989 Boundary-layer receptivity to long-wave free-stream disturbances. Ann. Rev. Fluid Mech. 21, 137-166.

Goldstein, M.E., Sockol, P.M. \& SAnZ, J. 1983 The evolution of Tollmien-Schlichting waves near a leading edge. Part 2. Numerical determination of amplitudes. J. Fluid Mech. 129, 443-453.

Hammerton, P.W. \& Kerschen, E.J. 1996a Boundary-layer receptivity for a parabolic leading edge: the small Strouhal number limit. To be submitted to J. Fluid Mech.

Hammerton, P.W. \& Kerschen, E.J. 1996b Leading-edge receptivity for bodies at angle-ofattack. To be submitted to J. Fluid Mech.

HeinRICH, R.A.E. 1989 Flat-plate leading-edge receptivity to various free-stream disturbance structures. PhD thesis, University of Arizona.

HeinRICH, R.A.E. \& Kerschen, E.J. 1989 Leading-edge boundary layer receptivity to various free-stream disturbance structures. Z. angew. Math. Mech. 69 6, T596-598.

Keller, H.B. \& CeBeCI, T. 1970 Accurate numerical methods for boundary layer flows- I. Two-dimensional laminar flows. In Proceedings of the Second International Conference on Numerical Methods in Fluid Mechanics (ed. M. Holt) pp. 92-100. Springer.

Kerschen, E.J. 1990 Boundary layer receptivity theory. Appl. Mech. Rev. 43, 152-157.

LAm, S.H. \& RotT, N. 1960 Theory of linearized time-dependent boundary layers. Cornell Univ. Grad. School of Aero. Engineering Rep. AFOSR TN-60-1100.

LAM, S.H. \& RotT, N. 1993 Eigen-functions of linearized unsteady boundary layer equations. J. Fluids Eng. 115, 597-602.

Libby, P.A. \& Fox, H. 1963 Some perturbation solutions in laminar boundary layer theory. Part 1: The momentum equation. J. Fluid Mech. 17, 433-449.

Lighthill, M.J. 1954 The response of laminar skin friction and heat transfer to fluctuations in the free-stream velocity. Proc. $R$. Soc. Lond. A 224 1-23.

Lin, N., ReED, H.L. \& SARIC, W.S. 1992 Effect of leading-edge geometry on boundary-layer receptivity to freestream sound. In Instability, Transition $\&$ Turbulence (eds: Hussaini, M.Y., Kumar, A., \& Streett, C.L.). Springer.

Murdoch, J.W. 1981 Tollmien-Schlichting waves generated by unsteady flow over parabolic cylinders. AIAA-81-0199.

Morkovin, M.V. 1969 Critical evaluation of transition from laminar to turbulent shear layers with emphasis on hypersonically traveling bodies. Air Force Flight Dyn Lab., WrightPatterson AFB, Ohio. AFFDL-TR-68-149.

Noble, B. 1958 Methods based on the Wiener-Hopf Technique. Pergamon.

Saric, W.S., Wei, W. \& Rasmussen, B. 1994 Effect of leading edge on sound receptivity. In Laminar-Turbulent Transition (ed. R. Kobayashi) pp. 413-420. Springer. 
SEdov, L.I. 1965 Two-dimensional problems in hydrodynamics and aerodynamics. Interscience Publishers.

VAN DyKe, M.D. 1964a Higher approximations in boundary layer theory. Part 3. Parabola in uniform stream. J. Fluid Mech. 19, 145-159.

VAN Dyke, M.D. 1964b Perturbation methods in Fluid Mechanics. Academic. 